\title{
REVIEW ARTICLE OPEN Paving the way towards an effective treatment for multiple sclerosis: advances in cell therapy
}

\author{
M. J. Mansilla ${ }^{1,2}$, S. Presas-Rodríguez ${ }^{3,4}$, A. Teniente-Serra ${ }^{1,2}$, I. González-Larreategui ${ }^{1,2}$, B. Quirant-Sánchez ${ }^{1,2}$, F. Fondelli $^{1,2}$, N. Djedovic $^{5}$, \\ D. Iwaszkiewicz-Grześ ${ }^{6,7}$, K. Chwojnicki ${ }^{8}$, Đ. Miljković ${ }^{5}$, P. Trzonkowski ${ }^{6,7}$, C. Ramo-Tello ${ }^{3,4}$ and E. M. Martínez-Cáceres ${ }^{1,2}$
}

Multiple sclerosis (MS) is a leading cause of chronic neurological disability in young to middle-aged adults, affecting $~ 2.5$ million people worldwide. Currently, most therapeutics for MS are systemic immunosuppressive or immunomodulatory drugs, but these drugs are unable to halt or reverse the disease and have the potential to cause serious adverse events. Hence, there is an urgent need for the development of next-generation treatments that, alone or in combination, stop the undesired autoimmune response and contribute to the restoration of homeostasis. This review analyzes current MS treatments as well as different cell-based therapies that have been proposed to restore homeostasis in MS patients (tolerogenic dendritic cells, regulatory T cells, mesenchymal stem cells, and vaccination with T cells). Data collected from preclinical studies performed in the experimental autoimmune encephalomyelitis (EAE) model of MS in animals, in vitro cultures of cells from MS patients and the initial results of phase I/II clinical trials are analyzed to better understand which parameters are relevant for obtaining an efficient cell-based therapy for MS.

Keywords: multiple sclerosis; cell-based therapy; tolerance; neuroprotection; autoimmunity

Cellular \& Molecular Immunology (2021) 18:1353-1374; https://doi.org/10.1038/s41423-020-00618-z

\section{MULTIPLE SCLEROSIS}

Multiple sclerosis (MS) is a chronic inflammatory and demyelinating disease that affects the central nervous system (CNS) and is characterized by inflammation, multifocal demyelination, axonal loss, and gliosis in both the white and gray matter. MS is a complex disease with considerable clinical and radiological heterogeneity. It was initially classified into four different phenotypes: relapsing-remitting MS (RRMS), secondary-progressive MS (SPMS), primary-progressive MS (PPMS), and relapsing-progressive MS (RPMS). ${ }^{1}$ RRMS $(85 \%)$ is characterized by acute relapses (acute or subacute episodes of new or increasing neurologic dysfunction in the absence of fever or infection) followed by remission with full or partial recovery. SPMS is defined as progressive clinical worsening over time after an initial relapsing course, with or without acute exacerbations during the progressive course. PPMS, accounting for $\sim 15 \%$ of MS, is characterized by clinical progression without relapse from disease onset. The term RPMS is used to describe the progressive accumulation of disability from onset with occasional relapses. This subtype is rarely diagnosed since it overlaps with other phenotypes in terms of its features.

In addition, since 2014, these phenotypes have also included the concept of "disease activity" based on clinical and MRI criteria in an effort to achieve better patient classification. ${ }^{2}$ For MS diagnosis, a combination of clinical, radiological, and laboratory criteria (presence of oligoclonal bands in the cerebrospinal fluid
(CSF)) is used. The most recent diagnostic criteria are the 2017 McDonald criteria. ${ }^{3}$

\section{MS pathogenesis}

Currently, the cause of MS remains unknown. In experimental autoimmune encephalomyelitis (EAE), an animal model of MS, myelin-specific T cells are believed to play a crucial role in its pathogenesis. ${ }^{4}$ In fact, the presence of circulating myelin-reactive T cells in MS patients has been extensively reported. However, the specific mechanisms that cause the activation and entrance of these cells into the CNS are still unknown. It has been postulated that a complex interaction between multiple genetic and environmental factors contributes to the dysregulation of peripheral immune homeostasis and the activation of autoreactive T cells. ${ }^{5}$

Several environmental factors, including infectious agents (mainly viruses), tobacco, diet (long-chain fatty acids, salt), gut microbiota, stress, sex hormones, and vitamin D deficiency, have been shown to be related to the triggering and development of disease. $^{5}$ The incidence of MS is higher in women than in men. MS symptoms often improve during late pregnancy, coinciding with high levels of estriol. ${ }^{6}$ In contrast, men are more prone to develop PPMS later in life, correlating with the physiological decline in testosterone with age. ${ }^{7}$

Extensive studies have been performed to understand the genetic contribution to MS, and more than 200 loci that promote a

\footnotetext{
${ }^{1}$ Division of Immunology, LCMN, Germans Trias i Pujol University Hospital and Research Institute, Barcelona, Spain; ${ }^{2}$ Department of Cellular Biology, Physiology and Immunology, Universitat Autònoma de Barcelona, Bellaterra, Spain; ${ }^{3}$ Multiple Sclerosis Unit, Department of Neurosciences, Germans Trias i Pujol University Hospital, Barcelona, Spain; ${ }^{4}$ Department of Medicine, Universitat Autònoma de Barcelona, Bellaterra, Spain; ${ }^{5}$ Department of Immunology, Institute for Biological Research "Siniša Stanković"- National

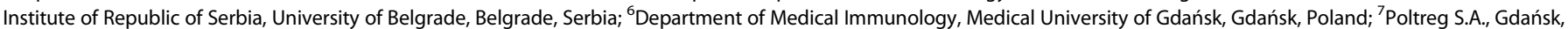
Poland and ${ }^{8}$ Department of Anaesthesiology \& Intensive Care, Medical University of Gdańsk, Gdańsk, Poland

Correspondence: M. J. Mansilla (mjmansilla@igtp.cat) or E. M. Martínez-Cáceres (emmartinez.germanstrias@gencat.cat)
}

Received: 13 July 2020 Accepted: 3 December 2020

Published online: 6 May 2021 
predisposition to MS have been identified, suggesting a complex disease etiology. Nearly all the gene regions identified so far contain genes involved in immune mechanisms. The major HLA$D R B 1^{*} 1501$ locus accounts for $30 \%$ of the overall risk. ${ }^{8,9}$ Moreover, emerging evidence indicates that the DNA methylome actively participates in gene $\times$ environment interactions, and several studies have shown an aberrant DNA methylome profile develops in MS. $^{10}$

Factors such as the presence of dysfunctional regulatory $T$ cells (Tregs) $^{11}$ or dendritic cells (DCs) ${ }^{12}$ and alterations in cytokine production may facilitate the entry of proinflammatory myelinspecific autoreactive T cells into the CNS (reviewed in ${ }^{13,14}$ ). In this context, IFN- $\gamma$-producing Th17.1 $\left(\mathrm{CCR}^{+}{ }^{+} \mathrm{CXCR}^{+}{ }^{+} \mathrm{CCR} 4^{-}\right)$cells have been identified as relevant in disrupting the permeability of the $\mathrm{BBB}$ in MS. ${ }^{15}$ Memory B cell precursors and IFN- $\gamma$-producing $\mathrm{CD}^{+}$ T cells also express high levels of CCR6, as they can enter the CNS. In addition to chemokine receptors and proinflammatory cytokines, adhesion molecules such as integrin a4 $\beta 1$ (VLA-4), which induces firm adhesion to vascular cell adhesion protein 1 (VCAM1) on brain endothelial cells, and activated leukocyte cell adhesion molecule enhance the transmigration of pathogenic $B$ and $T$ cell subsets. $^{16,17}$

As an alternative to the immune-mediated cause of MS, it has been postulated that CNS-intrinsic events (for example, CNS viral infection or processes leading to primary neurodegeneration) may trigger disease development, with the infiltration of autoreactive lymphocytes occurring as a secondary phenomenon. ${ }^{18,19}$

The brain and spinal cord are surrounded by the meninges, which provide first-line protection to the CNS. They are constituted by three layers: the dura mater, located directly under the skull or vertebral column; arachnoid mater; and pia mater, in close contact with the CNS parenchyma. The CSF drains through the subarachnoid space, an anatomical gap between the arachnoid mater and pia mater (both known as the leptomeninges). Interestingly, the existence of a rudiment of lymphatic vessels within the meninges was recently described, supporting the existence of a physical connection between the fluids, immune cells, and macromolecules of the CNS and the deep cervical lymph nodes, establishing physical contact between the CNS and the immune system. ${ }^{19,20}$

Most knowledge of MS pathogenesis has been obtained in the EAE model, the animal model of MS. This model is typically induced by either active immunization with myelin-derived proteins or peptides in adjuvant or by passive transfer of activated myelin-specific CD4+ T lymphocytes and reproduces most of the main clinical and histopathological characteristics of MS. Although this model has some limitations, as it is an induced model, it is a powerful tool to investigate MS pathogenesis and potential therapeutic strategies. ${ }^{21,22}$ In EAE, several days before inflammatory cells are detected in the CNS, an influx of peripherally derived immune cells within the meninges occurs. ${ }^{23}$ Myelin antigens that drain from the CNS to the meninges via the CSF are presented by infiltrating or resident antigen-presenting cells (APCs) (perivascular macrophages or resident APCs, e.g., microglial cells). ${ }^{24-26}$ This causes myelin-specific $T$ cell reactivation. Following myelin recognition by autoreactive pathogenic T cells, a complex immune response is produced, facilitated by the entrance of other cell types (B cells, NK cells, macrophages, and innate immune cells) and the production of proinflammatory cytokines and reactive oxygen and nitrogen species (ROS and RNS, respectively); this results in disruption of the blood-brain barrier (BBB) and the entrance of cells into the CNS parenchyma, leading to perivascular inflammation, demyelination, and neuronal damage. ${ }^{27}$

Interestingly, in contrast to $\mathrm{EAE}, \mathrm{CD}^{+} \mathrm{T}$ cells are found more frequently than $\mathrm{CD}^{+}$cells in acute and chronic MS lesions. CD8 ${ }^{+}$ $T$ cells directly attack oligodendrocytes (via the secretion of granzymes and perforin), causing apoptosis and damaging neurons via the release of cytolytic granules, leading to axonal dissection. ${ }^{28}$ Up to a quarter of $\mathrm{CD}^{+}{ }^{+}$cells in the active lesions of MS patients can produce IL-17 and are thought to be mucosaassociated invariant T (MAIT) cells. It has been suggested that these $\mathrm{CD}^{+}$cells, characterized by the expression of a semiinvariant T cell receptor (a dimer of Va7.2 with Ja12, Ja20, or Ja33), play an important role in disease pathogenesis. ${ }^{29}$

In recent years, the pathogenic role of B cells in MS has been highlighted. ${ }^{30}$ Clonally expanded $B$ cells can be found in the meninges, parenchyma and CSF. B cells produce antibodies intrathecally with an oligoclonal pattern (which can be observed by comparison with serum samples from the same patient). Ectopic lymphoid follicles, which sequester antigens and facilitate $B$ and $T$ cell activation, have been observed proximal to cortical demyelinating lesions in the meninges of MS patients, and their frequency correlates with disease severity. ${ }^{31-33}$ The presence of tertiary lymph follicles suggests that B cell maturation is sustained locally, contributing to the intrathecal synthesis of immunoglobulins. ${ }^{31,32}$ In addition to their roles in antigen presentation to Th1 and Th17 cells and antibody production, B cells secrete proinflammatory cytokines (e.g., IL-6, TNF, granulocyte-macrophage colony-stimulating factor (GM-CSF)), promoting CNS inflammation and demyelination. ${ }^{34}$

There is increasing evidence that innate immune cells (NK, DCs, macrophages, mast cells, and innate lymphoid cells (ILCs)), normal residents of the meninges, are involved in the pathogenesis of MS, affecting both its initiation and progression (reviewed by Brown et al. $\left.^{33}\right)$. Mast cell transcripts that encode mast cell-associated molecules, such as tryptase histamine or FceR1, have been observed in the demyelinating lesions of MS patients. ${ }^{35}$ They can increase BBB permeability, contributing to the initiation of chronic inflammation. ${ }^{36}$ Moreover, interactions between resident mast cells and autoreactive $T$ cells in the meninges induce caspase-1-dependent IL-1 $\beta$ production by mast cells, activating GM-CSF production by T cells. ${ }^{37,38} \mathrm{GM}-\mathrm{CSF}$ is an essential growth factor for T cell encephalitogenicity, inducing the recruitment of $\mathrm{CCR2}^{+}$inflammatory monocytes into the CNS. ${ }^{39-42}$ Additionally, TNF expression by mast cells is essential for the early recruitment of neutrophils to the meninges and CNS. ${ }^{23,36,43}$ Neutrophil products, i.e., proteolytic enzymes, including matrix metalloproteinase 9, ROS and structures composed of DNA and proteins called neutrophil extracellular traps, damage the $B B B$, thus supporting a role for neutrophils in MS pathogenesis. ${ }^{43-45}$

CD45 ${ }^{+}$Lin-IL-7Ra ${ }^{+}$ILCs constitute a heterogeneous group of innate immune cells that have more recently been related to MS pathogenesis: group 1 (ILC1), group 2 (ILC2), and group 3 (ILC3) cells, analogous to Th1, Th2, and Th17 cells, respectively. These cells mostly remain in tissues and exert their effects locally. ${ }^{46}$ Similar to Th cells, ILCs can also exhibit considerable phenotypic plasticity. ${ }^{46}$ Each group of ILCs plays a different role in EAE-related inflammatory responses. ILC2s express ST2, the heterodimeric IL33 receptor. Upon activation, these cells produce the Th2 cytokines IL4, IL-5, IL-9, and IL-13. ${ }^{47}$ IL-33 induces ST2 ${ }^{+}$ILC2s to produce IL-13 and other Th2-polarizing cytokines, which in turn promote a nonpathogenic Th2-dominated response. In contrast, ILC1s and ILC3s produce IFN- $\gamma$, IL-17, GM-CSF, and other cytokines that have been linked to EAE pathogenesis. Interestingly, the lymphoid tissue inducer subset of ILC3s drives ectopic lymphoid follicle formation. ${ }^{48}$

Natural killer (NK) cells have been widely studied in the context of MS and EAE and assigned both pathogenic and protective roles (reviewed in $^{49,50}$ ). CD56 ${ }^{\text {dim }}$ cells, the major cytotoxic NK population, can kill oligodendrocytes, astrocytes, and microglia in vitro. In contrast, regulatory CD56 ${ }^{\text {hi }}$ NK cells produce neurotrophic factors such as brain-derived neurotrophic factor (BDNF) and neurotrophin-3, consistent with a role in neural repair.

Additionally, CNS-resident cells (mainly microglia) are highly sensitive to homeostatic disturbances and can produce neurotoxic inflammatory mediators (cytokines, chemokines, and ROS), 
promoting and sustaining neurodegeneration (reviewed $\mathrm{in}^{13}$ ). Importantly, astrocytes are key players recruiting lymphocytes and inducing the innate response during the early stage of white matter lesion formation. In contrast, astrocytes can also restrict inflammation through scar formation and trigger neuroprotection and tissue repair. ${ }^{51}$ Regulatory B cells (Bregs), T cells (CD4 ${ }^{+} \mathrm{FoxP}^{+}$ (Tregs), $\mathrm{CD} 4^{+} \mathrm{Tr}^{+}$and CD8 Tregs), tolerogenic DCs (tolDCs) and regulatory $\mathrm{CD} 56^{\text {hi }}$ NK cells can regulate effector $T$ cells in the periphery or CNS through different mechanisms. It has been postulated that either a defect in the regulatory function of these cells or the increased resistance of effector T cells to suppressive mechanisms contributes to the pathogenic function of autoreactive T and B cells in MS. Alternatively, the dysfunction of peripheral regulatory cells could be indirectly driven by the dysregulation of tolerogenic APCs (reviewed in ${ }^{13}$ ).

\section{Neuropathology of MS}

In contrast to the increased inflammation of relapsing forms of MS neurodegenerative events are more severe in progressive MS. Nevertheless, there is no clear distinction between progressive and relapsing MS; both pathobiologies can occur during the disease.

Comparison of PPMS and SPMS reveals some quantitative differences in the presence of focal and active classical white matter lesions and in the global degree of inflammation, which is lower in PPMS than in SPMS. ${ }^{52}$ These differences can be explained by the two types of inflammation in MS patients (Fig. 1). During disease progression, patients can exhibit both types of inflammation, although the composition can be altered during relapses and progressive periods.

During the acute and relapsing phases of the disease, in which leakage of the BBB occurs, the major players are $\mathrm{T}\left(\mathrm{CD}^{+}\right.$and $\mathrm{CD}^{+}$) and $B$ lymphocytes that attack myelin, leading to demyelination. $\mathrm{CD} 8^{+}$cells have the phenotype of tissue-resident memory cells. $\mathrm{CD}^{+}{ }^{+}$cells proliferate focally and show signs of activation or clonal expansion, indicating local antigen recognition. The pathogenic role of $B$ cells is inferred by the beneficial effects observed after anti-CD20 therapies. However, B cells are thought to play different roles depending on their stage of differentiation or the activity stage of the lesions. Plasmablasts and plasma cells within MS lesions express high levels of IL-10, suggesting that they may ameliorate inflammation.

Inflammatory infiltrates may lead to focal areas of primary demyelination with variable axonal injury, which is mainly carried out by activated microglia and macrophages. Antibodies that recognize oligodendrocytes or astroglia may contribute to MS pathogenesis at this stage. After the initial autoimmune attack, lymphoid cells in the parenchyma undergo apoptosis, and macrophages and microglia can switch to an anti-inflammatory/ reparative phenotype.

In the progressive phases of MS, the second pattern of inflammation predominates. Leakage of the BBB is less pronounced, and $T$ and $B$ lymphocytes slowly accumulate in the connective tissue spaces of the brain and spinal cord, affecting the meninges and periventricular spaces. Infiltrating cells form focal aggregates resembling tertiary lymph follicles. $\mathrm{CD} 8^{+}$cells have the phenotype of tissue-resident memory cells with focal activation. Most cells of the B cell lineage in chronic lesions are plasmablasts and plasma cells. Tissue injury may at least be partly mediated by microglia and macrophage activation, oxidative injury and mitochondrial damage. This inflammation is associated with the expansion of pre-existing lesions, as well as diffuse neurodegeneration in normal-appearing white or gray matter. Interestingly, it has been found that this second type of inflammation is already present in the early stages of MS, after which it increases gradually with disease duration and patient age. ${ }^{52}$ The inflammation induced by peripheral immune cell infiltration and CNS-resident innate immune cells may contribute to acceleration of the aging processes in the CNS, followed by pronounced progressive neurodegenerative decline (reviewed $\mathrm{in}^{13}$ ).

\section{CELL-BASED TOLEROGENIC THERAPIES}

Targeting the fundamental cause of autoimmunity, i.e., the loss of tolerance to self-antigens, will provide the next steps forward to avoid the general immunosuppression induced by current treatments. Accumulating knowledge on the mechanisms of immune tolerance and activation has led to the development of tolerance-inducing cellular therapies with the specific objective of limited unwanted immune reactions over the long term (reviewed $i^{54}$ ). Phase I clinical trials using Tregs, mesenchymal stem cells (MSCs), or tolerogenic antigen-presenting cells, such as tolDCs and regulatory macrophages, for the treatment of autoimmune diseases (ADs) and prevention of transplant rejection have expanded in recent years. The results have confirmed so far that these cellular therapies are safe, with no relevant side effects, and well tolerated by patients. ${ }^{54}$ In this context, several cellular products have been developed for MS (Fig. 2), and phase I clinical trials are currently ongoing or finished. To advance the use of these therapies in the clinic, we should analyze the results and address remaining challenges, such as the optimal dose, administration route, frequency of administration, antigen specificity, and biomarkers of clinical and biological response, to design the next generation of clinical trials.

Tolerogenic dendritic cells

DCs are key players in controlling the immune response. They are highly efficient APCs that are able to activate the immunogenic T cell response and suppress it by inducing regulatory mechanisms. ${ }^{55}$ TolDCs are defined as semimature DCs with an intermediate phenotype between the phenotypes of immature (iDCs) and mature DCs (mDCs). It is not clear whether tolDCs constitute a different DC subset by themselves. ToIDCs are characterized by one or more of these features: the expression of low levels of costimulatory molecules (i.e., CD80, CD86, and CD40) and MHC class II, a reduced capacity to produce proinflammatory cytokines, upregulated expression of inhibitory molecules such as PDL1, ILT3 and ILT4 and secretion of immunoregulatory cytokines and mediators (IL-10, TGF- $\beta$, IDO, heme oxygenase- 1 or FasL). ${ }^{56,57}$ Because of the semimature or mature resistance phenotype of tolDCs, the proper activation of $\mathrm{T}$ cells by costimulatory molecules and proinflammatory cytokines following antigen recognition is limited. Under these conditions, instead of inducing the activation and clonal expansion of $\mathrm{T}$ cells, tolDCs promote $\mathrm{T}$ cell hyporesponsiveness that is mainly mediated by the induction of T cell anergy, T cell depletion triggered by apoptosis induction or the induction of Treg differentiation (reviewed $\mathrm{in}^{58,59}$ ).

Importantly, tolDCs can be generated ex vivo from autologous human peripheral blood monocytes. Indeed, over the last 20 years, a wide variety of protocols describing the induction of tolDCs with several stimuli, such as anti-inflammatory cytokines (IL-10 and TGF- $\beta$ ), pharmacological agents and immunosuppressant compounds (rapamycin, different corticosteroids, vitamin D3, aspirin, mitomycin C, and the NF-KB inhibitor BAY11-7082), and other strategies, such as genetic engineering for the selective repression or induction of key molecules and pathways, among many others, have emerged (reviewed $i^{54,58}$ ). Most of these protocols share several features, such as the differentiation of monocytes in the presence of GM-CSF and IL-4, as well as the addition of a maturation stimulus (which usually consists of different combinations of LPS, monophosphoryl lipid A, TNF, IL-1 $\beta$, prostaglandin E2 (PGE2) and IL-6), to maintain tolDCs in an activation-resistant state, as this is an absolute requirement for tolDC therapy.

Analysis of preclinical data for the evaluation of tolDC treatment in $E A E$, as well as in vitro studies on peripheral blood leukocytes 


\section{Relapsing MS $:$ Progressive MS}

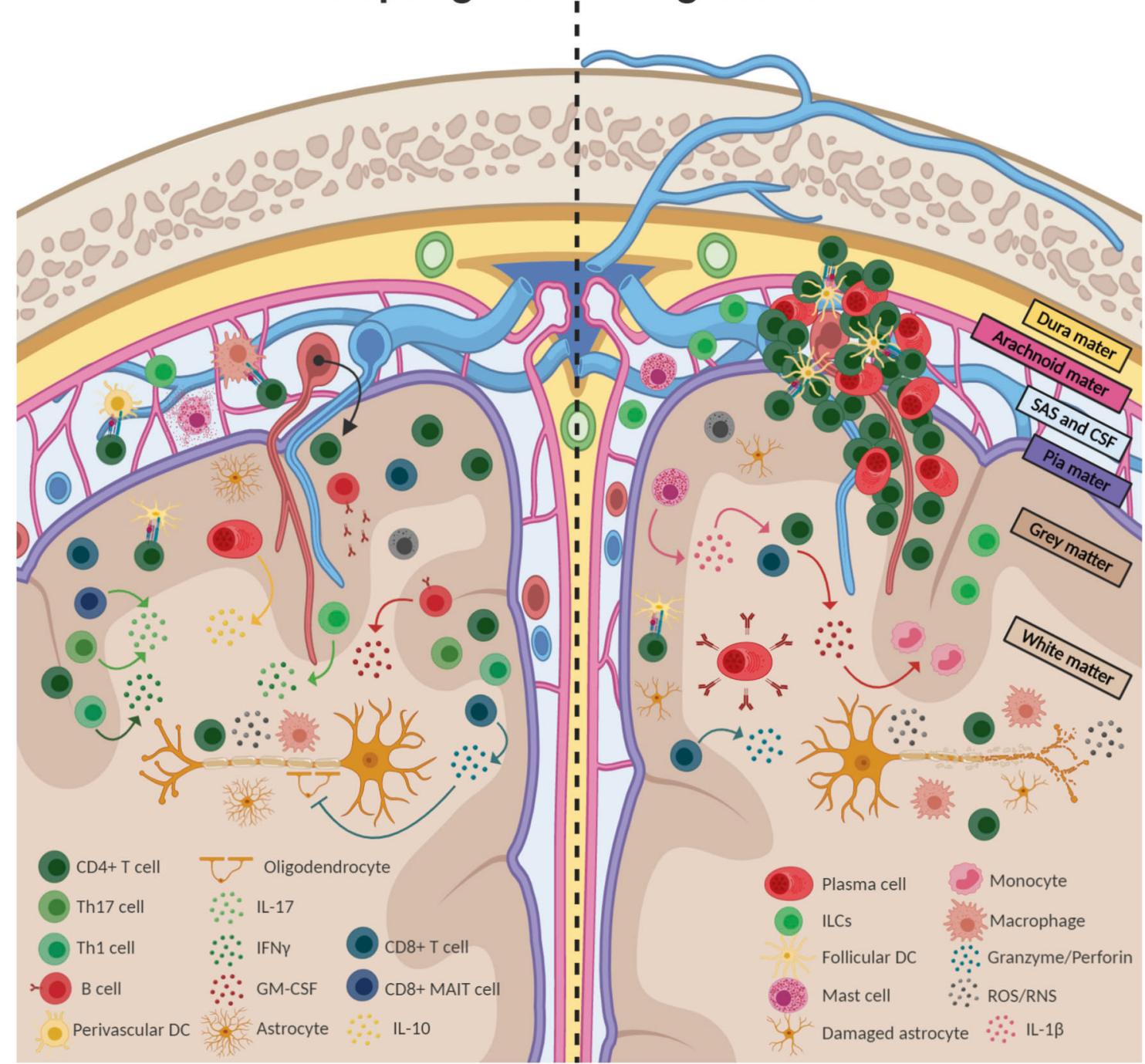

Fig. 1 Multiple sclerosis pathogenesis in both relapsing and progressive disease. Scheme representing the major cells and molecules that play a role in the two different stages of MS. The dashed line allows comparison of the differences between relapsing and progressive MS. Arrows indicate release, while inhibitors indicate inhibition. The black arrowhead on a dotted line indicates transmigration. CSF cerebrospinal fluid, DC dendritic cells, GM-CSF granulocyte-macrophage colony-stimulating factor, IFN interferon, IL interleukin, ILCs innate lymphoid cells, MAIT cells mucosal-associated invariant T cells, MS multiple sclerosis, RNS reactive nitrogen species, ROS reactive oxygen species, SAS subarachnoid space, Th T helper

from MS patients (Table 1), is crucial to better understand the requirements and characteristics for the design of efficient tolDC products for MS patients.

Antigen specificity. Several studies in the EAE model of MS have demonstrated that clinical efficacy of tolDCs is achieved only when the cells are loaded with disease-related autoantigens ${ }^{60-71}$ (Table 1). Although the etiology of MS is unknown, it is widely accepted that it is an autoimmune-mediated disease directed against several myelin proteins, such as MBP, proteolipid protein (PLP), and myelin oligodendrocyte glycoprotein (MOG). Consequently, when thinking about the translation of tolDC therapy to humans, the loading of a single antigen on tolDCs is unlikely to be sufficiently effective. Moreover, due to the epitope spreading phenomenon, the same patient may also exhibit extended reactivity to additional myelin epitopes following disease progression. Therefore, in tolDC phase I clinical trials for MS patients, a cocktail of myelin peptides containing the most relevant autoreactive MOG, PLP, and MBP peptides seems to be a better strategy. ${ }^{72,73}$ In fact, the three phase I clinical trials of peptideloaded toIDCs in MS have employed a similar approach using a pool of myelin peptides (NCT02283671, NCT02903537, and NCT02618902) (Table 2).

Dose, timing, and frequency. Although studies in animal models are extremely useful, extrapolation of the dose, timing, and frequency of administration to patients is not straightforward. From publications using different tolDC types to treat $E A E$, i.v. administration of a million antigen-specific tolDCs has demonstrated promising results in reducing EAE severity (Table 1). However, extrapolation of the dose from these studies is not feasible. The monocyte-derived tolDCs recovered after the in vitro differentiation process number a maximum of several million, depending on the patient. Consequently, thus far, direct extrapolation of toIDC doses from EAE studies is not feasible. In fact, in a phase I trial of i.v. dexamethasone-tolDC (dexa-tolDC) 


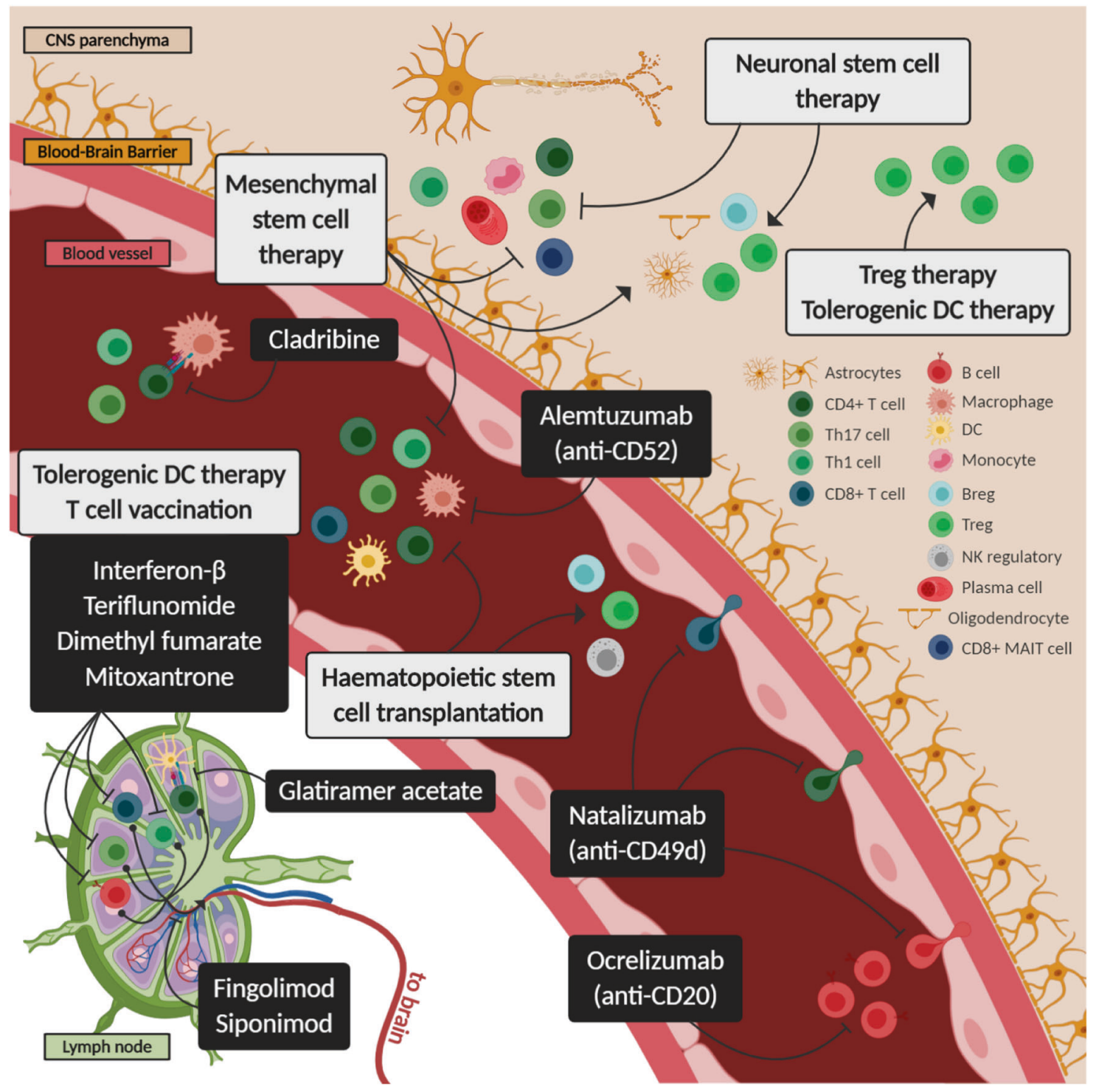

Fig. 2 Proposed mechanisms of action of approved treatments for multiple sclerosis and cell-based therapies. Representation of the mechanisms of action of current treatments (black boxes) and cell-based therapies (gray boxes). Arrows indicate induction, while inhibition symbols indicate inhibition. Breg regulatory B cells, CNS central nervous system, DC dendritic cells, MAIT cell mucosal-associated invariant T cells, NK natural killer cells, Th T helper, Treg regulatory T cells

administration, a technical limitation was reported related to reaching $300 \times 10^{6}$ cells. $^{74}$ Since cells administered intravenously exhibit a wide biodistribution of cells (meaning that a large number of tolDCs would be necessary to reach the secondary lymphoid organs), other routes of administration are currently being tested by our group and others (Table 2). This issue will be discussed below.

As shown in Table 1, many of the EAE studies analyzed the efficacy of tolDCs before the onset of clinical symptoms (prophylactic or late prophylactic administration). In contrast, other authors have attempted to reproduce the real-world situation of MS patients for therapeutic administration. ${ }^{61,66,67,70}$ From those studies, antigen-specific VitD3-tolDCs demonstrated a remarkable therapeutic effect. ${ }^{61,66,67}$

Focusing on therapeutic studies in EAE, the administration of at least 3 doses of antigen-specific tolDCs treated with VitD3 or BD750 (a JAK3/STAT5 inhibitor) in the early stage of disease abrogated disease progression. ${ }^{61,66,67,70}$ Considering translation to MS, the use of multiple tolDC injections seems optimal. However, this would imply repeated production of tolDC batches (which would require several rounds of leukapheresis and in vitro differentiation under good manufacturing practice conditions). Hence, the use of cryopreserved cells is a feasible option. ${ }^{6,75}$ In fact, at least for VitD3-tolDCs, both human and murine cryopreserved cells retain phenotypical and functional tolerogenic characteristics. Importantly, therapeutic administration of cryopreserved MOG $_{40-55}$-VitD3-tolDCs in EAE mice showed clinical efficacy, reducing $T$ cell autoreactivity and triggering the generation of FoxP3 $^{+}$Tregs in vivo. ${ }^{6}$ All these data were comparable with those obtained using fresh cells. ${ }^{67}$ To go one step further, we explored the effect of long-term treatment with cryopreserved $\mathrm{MOG}_{40-55^{-}}$ VitD3-tolDCs in EAE. Following 3 therapeutic administrations of $\mathrm{MOG}_{40-55}$-tolDCs every 4 days, additional doses were administered when the mean clinical score of the group increased. Strikingly, we observed that periods of long-lasting clinical stability increased progressively. Immunological examination of mice revealed increased proportions of Bregs and activated NKT cells as well as a reduction in immunogenic NK cells in the spleens of treated mice, which might explain (at least in part) the beneficial effect of $\mathrm{MOG}_{40-55}$-tolDCs. 


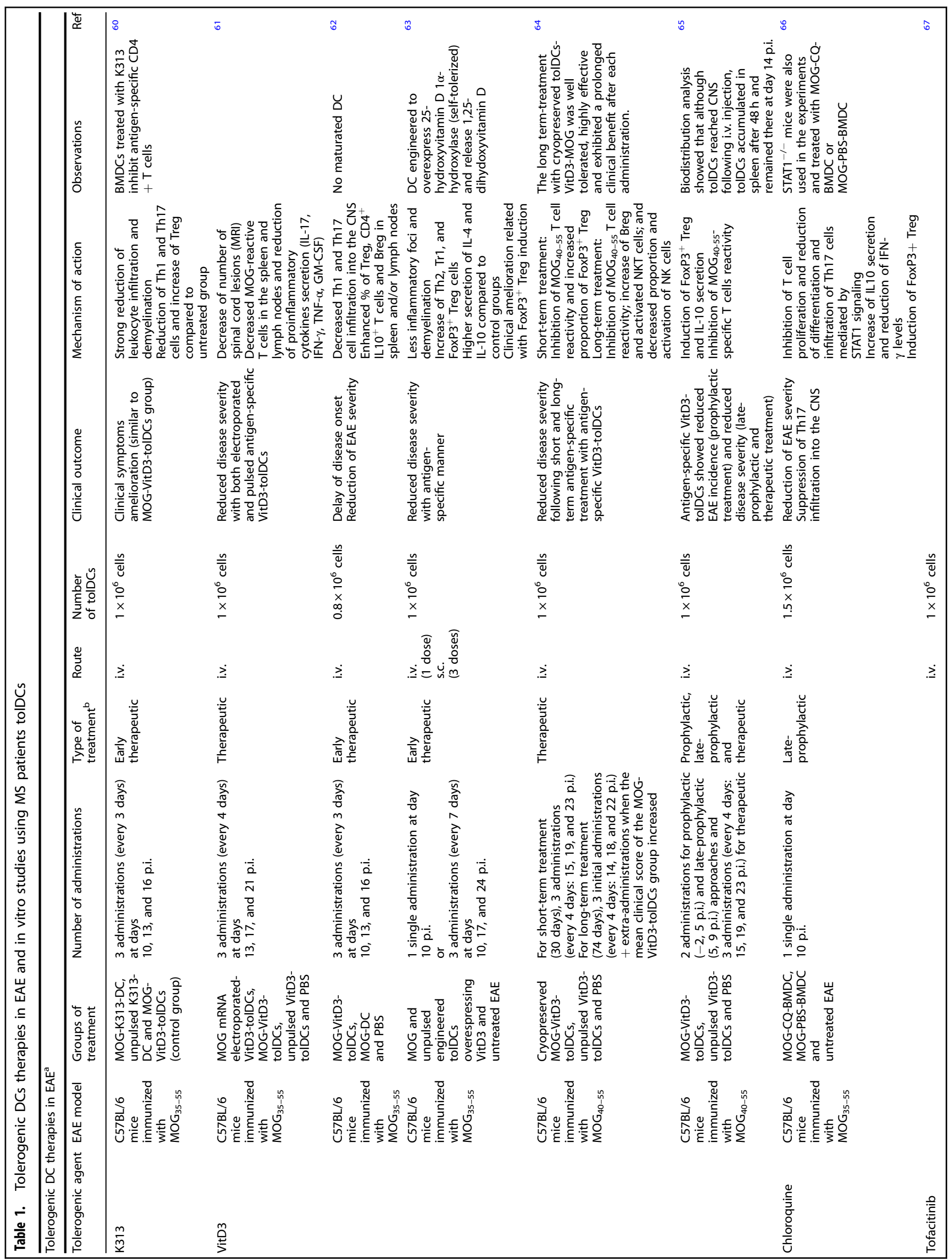




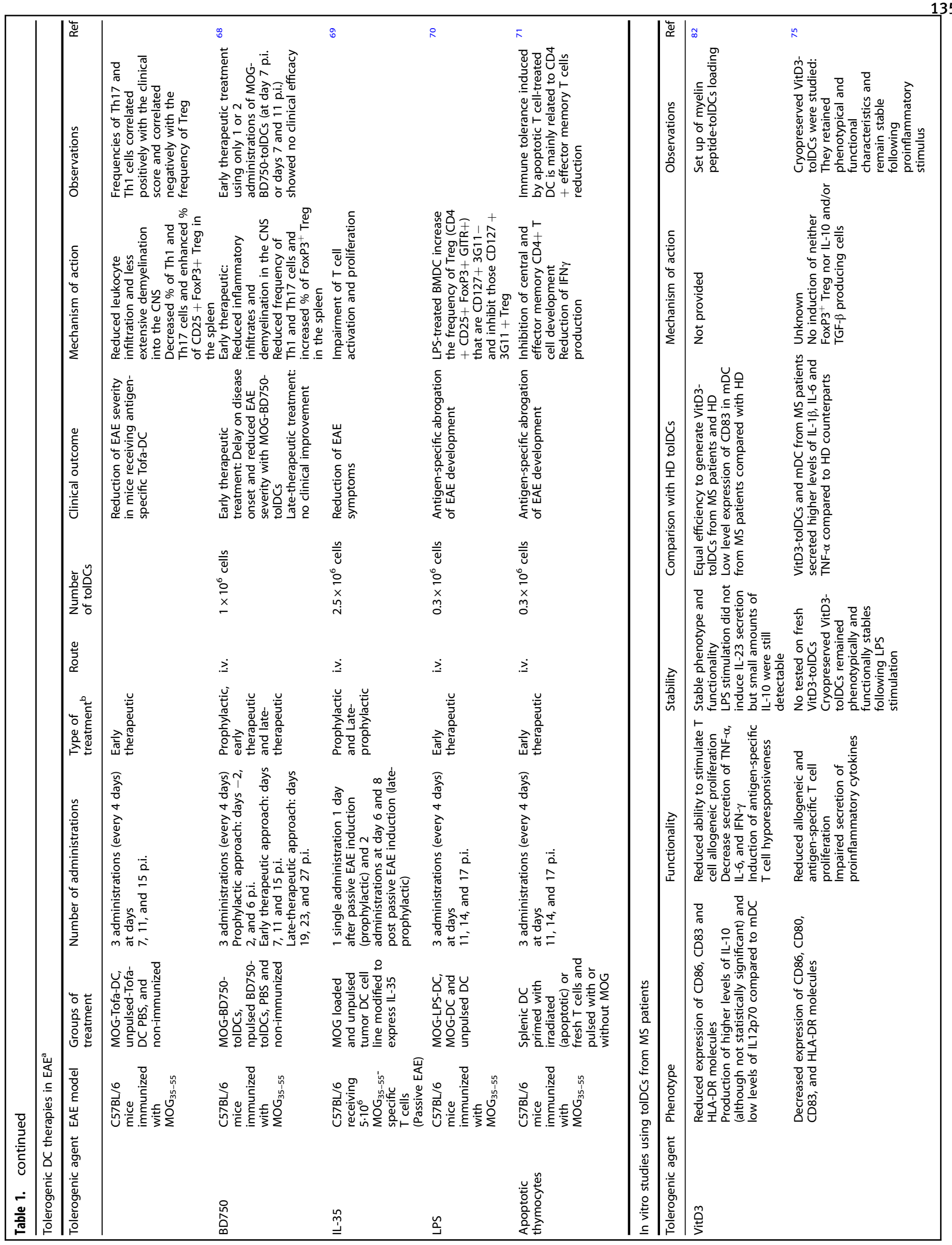


The effect of tolDCs in the chronic phase of EAE has been analyzed using $\mathrm{MOG}_{35-55}-\mathrm{BD} 750$-tolDCs. Unfortunately, no clinical benefits were observed even though 3 doses of $\mathrm{MOG}_{35-55}$-tolDCs were administered every 4 days. ${ }^{70}$ Although more studies are needed, these results suggest that toIDC therapy should be used in the first stages of the disease.

Route of administration. The safety of i.v., intraperitoneal (i.p.), intradermal (i.d.), intranodal (i.n.) and even intra-articular routes of human toIDC administration has been demonstrated in different phase I clinical trials. ${ }^{74,76-79}$ The route of administration is crucial due to two important issues. On the one hand, the treatment must promote tolerogenic in vivo effects. In this context, i.v. administration is considered the most tolerogenic route of administration, showing tolerogenic effects superior to those of i.d. administration in nonhuman primates. ${ }^{80}$ On the other hand, the selected route of administration must allow tolDCs to reach the draining lymph nodes or inflamed tissues. In this regard, either i.d. administration near the draining lymph nodes or direct i.n. tolDC injection could be an alternative to i.v. administration. Interestingly, two coordinated phase I clinical trials using VitD3-tolDCs will compare these two routes of administration ${ }^{81}$ (Table 2).

Mechanism of action. The mechanism of action of several types of tolDCs has been analyzed. ${ }^{54}$ Most tolDCs impair $T$ cell alloproliferation in in vitro cultures. Moreover, they also act through different pathways. Dexa-tolDCs produce IL-10, tolDCs generated in the presence of exogenous IL-10 (DC-10) induce IL10-producing Tregs ( $\mathrm{Tr} 1$ cells), and VitD3-tolDCs induce $\mathrm{T}$ cell hyporesponsiveness in an antigen-specific manner without affecting the ability of other $\mathrm{T}$ cells to respond to unrelated antigens. $^{75,82}$ In addition, a transcriptomic analysis of autologous $\mathrm{CD}^{+}{ }^{+}$T-cells primed with antigen-specific VitD3-tolDCs revealed profound genetic downregulation, mainly affecting factors related to the cell cycle and proinflammatory immune response processes. ${ }^{83}$

Dexa+VitD3-tolDCs regulate $\mathrm{CD}^{+}{ }^{+} \mathrm{T}$ cell cytokine production in RA patients in a TGF- $\beta 1$-dependent manner. ${ }^{84}$ Additionally, TGF- $\beta$ secreted by tolDCs is an important immunoregulatory mediator involved in the induction of Tregs. Interestingly, tolDCs differentiated in the presence of low doses of GM-CSF and in the absence of IL-4, referred to as autologous tolerogenic dendritic cells (ATDCs), have the capacity to reduce $T$ cell proliferation via a novel mechanism involving lactate secretion. ${ }^{85}$ Lactate secreted by ATDCs exerts its immunosuppressive effect by downregulating $T$ cell glycolysis. Currently, the specific mechanisms triggered by different tolDCs in vivo remain elusive. Inhibition of antigen-specific $T$ cell proliferation, increases in FoxP3 ${ }^{+}$Treg numbers, decreases in proinflammatory Th17 and Th1 cell numbers in both the periphery (spleen and lymph nodes) and CNS, and increased levels of IL-10 have been described ${ }^{60-65,67-70,75,82,84,86,87}$ (Table 1). Interestingly, an increased frequency of Bregs was found after MOG-VitD3-tolDC therapy in $\mathrm{EAE}^{64,66}$ similar to the results of the first phase I clinical trial with genetically modified tolDCs conducted by Giannoukakis et al. in patients with type I diabetes. ${ }^{77}$ In MS, an increase in IL-10 production by PBMCs isolated from treated patients was described. $^{74}$ In addition, tolDCs can induce the secretion of indoleamine 2,3-dioxygenase (IDO), a potent regulatory enzyme that catalyzes the degradation of tryptophan required for $T$ cell proliferation. ${ }^{88}$ Altogether, these data indicate that tolDC therapy can trigger a complex tolerogenic immune cascade, with anergy or elimination of pathogenic Th1/Th17 cells and induction of regulatory cells (FoxP3 ${ }^{+}$Tregs, $\operatorname{Tr} 1$ cells, and Bregs), that can reduce EAE severity, even in mice with established clinical signs of paralysis.

Phenotype, function, and stability. Currently, no common biomarkers of tolerogenic function in different types of monocytederived tolDCs have been identified. ${ }^{89}$ Thus, their phenotypic, as well as functional, characterization requires comparison with 
mDCs generated in parallel to certify their correct production before administration to patients. In addition, the stability of tolDCs is crucial, and stability can be analyzed in vitro in tolDCs exposed to a proinflammatory milieu to ensure that there is no conversion of tolerogenicity to immunogenicity. ${ }^{90}$ No data regarding the stability of these cells in vivo after their administration to patients have been obtained so far. In this context, it is expected that the use of cell trackers will provide relevant information to better understand the in vivo mechanism of action of tolDCs. ${ }^{91}$

Clinical trials in MS. To the best of our knowledge, a total of three phase I clinical trials using tolDCs for the treatment of MS patients are ongoing or have recently finished (Table 2 ). In one doseescalation phase $\mathrm{Ib}$ clinical trial, patients with MS $(n=8)$ and neuromyelitis optica $(n=4)$ received 3 i.v. injections $(50,100,150$, or $300 \times 10^{6}$ tolDCs in total) as three independent doses administered every 2 weeks. However, the last group did not receive the planned doses due to a technical limitation in obtaining the required number of cells. Clinically, the treatment was safe and well tolerated. ${ }^{74}$

Two coordinated phase I clinical trials in MS patients treated with autologous VitD3-tolDCs loaded with myelin peptides are currently ongoing simultaneously in Belgium and Spain in the context of the European $\mathrm{H} 2020$ framework. ${ }^{81}$ Both studies tested the safety and tolerability of autologous peptide-loaded VitD3tolDCs in a dose-escalation study using 5, 10, and $15 \times 10^{6}$ VitD3tolDCs/administration, with the first four of six independent doses administered every 2 weeks and the last two administered every 4 weeks. In addition, exhaustive immunomonitoring is being performed (Table 2).

Regulatory T cells

Since Treg induction is one of the most relevant and consistent mechanisms to achieve immunoregulation, cell therapy administering Treg cells is one of the most promising strategies that has been extensively investigated worldwide. ${ }^{92}$

Mechanism of action. Tregs in the body comprise the naturally occurring/thymic (tTreg) and induced/peripheral (pTreg) compartments. The latter compartment is further divided into several subsets, with Tr1 cells and Th3 cells being the main representatives. While tTregs are specifically designed to regulate the immune response from the progenitor stage in the thymus, pTregs are generated via the conversion of conventional $\mathrm{CD}^{+}$ $\mathrm{T}$ cells in the periphery during the immune response. ${ }^{93}$ Both subsets are efficient regulators of the immune response, but their origins suggest somewhat different activities. Thymic Tregs are anergized towards self-antigens in the thymus. These cells migrate to a site of inflammation and the local lymphoid tissue surrounding it and limit the immune response when selfantigens are sensed. In this way, tTregs protect the body from possible autoreactivity. This suppression is very precise, occurring mainly locally via cell-to-cell interactions with other cells that take part in the immune response. The main receptor of tTregs is CTLA4 (cytotoxic $T$ lymphocyte antigen 4, CD152), which links to receptors from the B7 family on APCs and limits the presentation of autoantigens. Surface TGF- $\beta$ and LAG-3 are involved in the suppression of NK cells, and PD-1/PD-L coupling is involved in the suppression of T cells and B cells. High expression of CD25 (IL2Ra subunit) allows tTregs to preferentially take up the majority of available IL-2, which triggers apoptosis of overactivated effector $T$ cells (conventional $T$ cells, Tconvs) in the surrounding microenvironment. This suppression is not limited to self-antigens, as the interaction with APCs can result in so-called "linked suppression". Specifically, when APCs present tolerized autoantigens with some alloantigens, tTregs interacting with APCs can also impose tolerance towards the alloantigen. This is an important advantage of the polyclonal preparation of tTregs used in cell therapy. The fact that pTregs arise in the periphery during immune responses, mainly from naïve $\mathrm{CD} 4^{+} \mathrm{T}$ cells, implies that they are specific to the antigen that triggers the immune response. The main regulatory mechanism of pTregs involves the secretion of suppressive factors such as IL-10, produced mainly by $\operatorname{Tr} 1$ cells, or TGF- $\beta$, produced by Th3 cells. ${ }^{92,94}$ Currently, tTregs are the main subset used in the clinic, but a limited number of trials using $\operatorname{Tr} 1$ cells have also been conducted.

Phenotype, function, and stability. Expression of the transcription factor FoxP3 is currently the main marker of Tregs. ${ }^{93}$ CD127 (the IL7 receptor) is negatively correlated with FoxP3. ${ }^{11,95}$ Importantly, mutations that render FoxP3 inactive in humans are responsible for immune dysregulation, polyendocrinopathy, enteropathy, and X-linked inheritance (IPEX). Nevertheless, the phenotype should always be confirmed with the suppressive function. Throughout their development from the progenitor stage to the immune response, tTregs undergo many changes that affect their activity. For example, there is a developmental link between Th17 cells and tTregs, which implies the plasticity of cells of these phenotypes. ${ }^{96}$ This is important for the stability of Tregs not only during in vitro manufacturing but also when tTregs are expected to function in the body. The stability of Tregs is also substantially affected by epigenetic changes in mature cells. For example, methylation of the Treg-specific demethylated region within the foxp3 gene significantly impairs suppressive function. ${ }^{97}$ Additionally, the inflammatory cytokine milieu can counteract the suppressive effects of tTregs, which should be considered when therapies with tTregs are designed. For example, TNF secreted by inflammatory cells can abrogate the suppressive effects of tTregs. ${ }^{98}$ This fragile phenotype should be taken into account during the manufacturing of Tregs for clinical applications, since trivial factors such as the time or temperature of expansion might affect the final activity of the cellular product. $^{99,100}$

Therapy with Tregs in EAE. While animal studies in many cases assume very early intervention, when there is little or no damage to the CNS, therapy in humans usually starts when the pathology is very advanced, as the first symptoms often occur only then. Likewise, the burden of inflammation and the location of the inflammatory process may be different between an animal model and humans in a trial, and this needs to be considered when translating the results into the clinic. One variable to consider is the time when the treatment is administered. In this context, analysis of different studies on Tregs shows that their administration in EAE was performed at different times: prophylactically (before or with immunization), late prophylactically (before the first clinical signs appeared), therapeutically (with the first clinical signs), and late therapeutically (5-8 days after the initial clinical signs). Details of the studies are given in Table 3. Here, we will focus on studies in which Tregs are administered therapeutically, as they more closely resemble the potential application of Tregs in MS.

Stephens et al. administered Tg4 CD25+CD62L hi MBP(Ac1-9)reactive Tregs to Ac1-9 peptide-immunized B10.PL or B10.PL $\times$ SJL mice at 18 days post immunization (d.p.i.). ${ }^{101}$ This was the time of remission from the first EAE relapse. While mice that were not treated with Tregs developed more relapses and chronicity of EAE in the subsequent days, the severity of EAE relapse was markedly reduced in Treg-treated mice. The effect was more pronounced in B10.PL x SJL mice, as Treg-treated mice were nearly disease-free at the end of the observation period (100 d.p.i.). These results imply that Treg application could be efficient in MS, even at the later stages of the disease when the vicious cycle of autoimmune reactivity is well established. In another study, Fransson et al. used Tregs derived from $\mathrm{CD}^{+} \mathrm{T}$ cells that had been modified with a lentiviral vector system to express a chimeric antigen receptor 


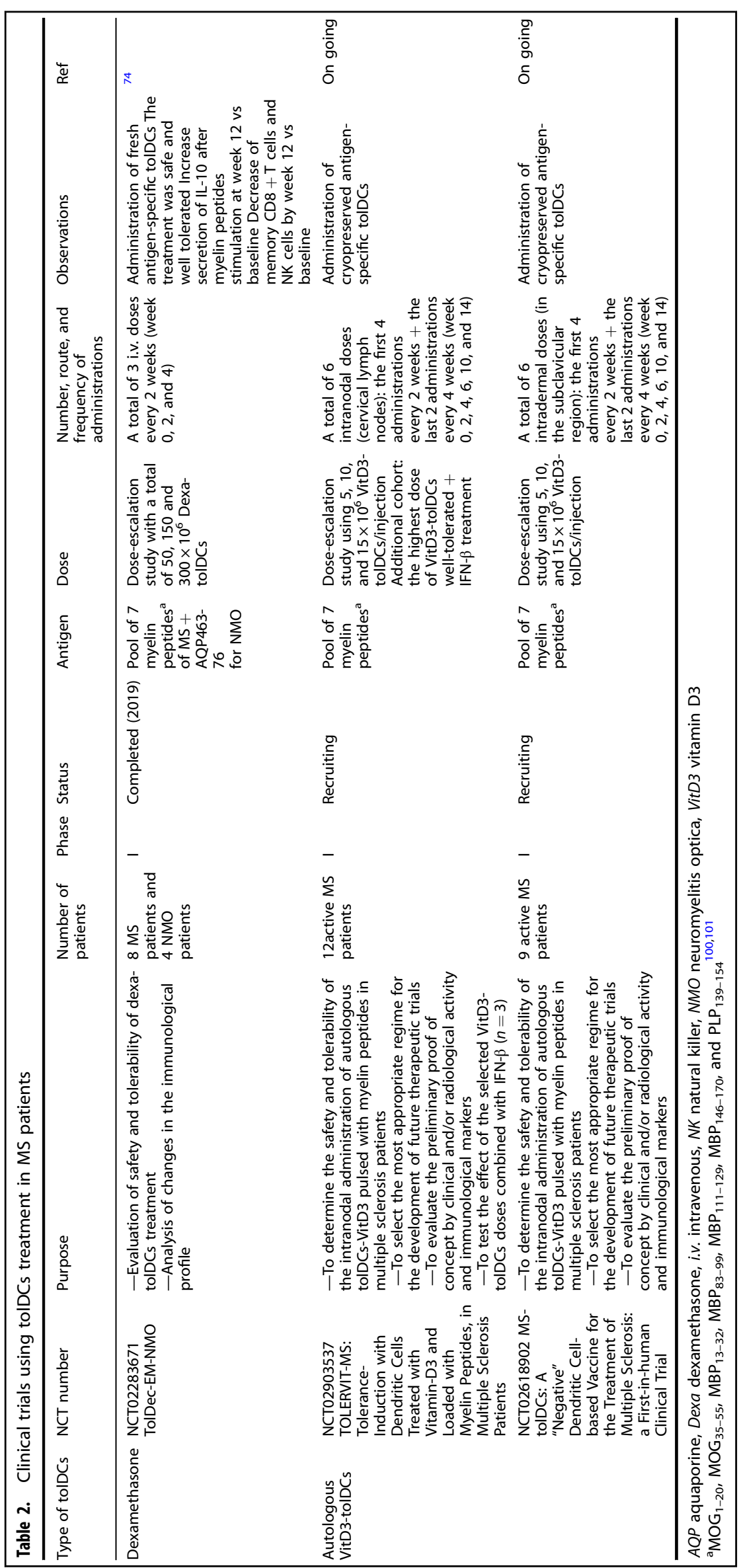




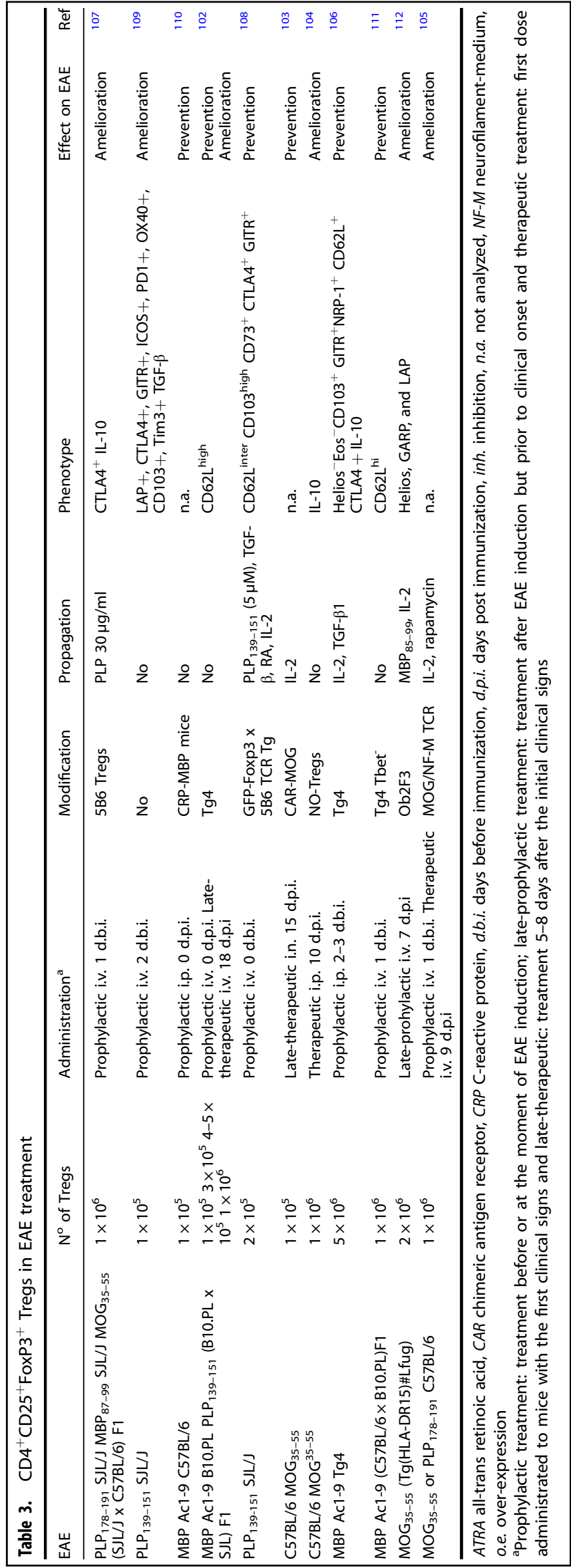

(CAR) targeting MOG in trans with the FoxP3 gene. They were able to produce Tregs with a strong affinity for MOG and persistent FoxP3 expression. ${ }^{102}$ Tregs were applied to C57BL/6 mice with $\mathrm{MOG}_{35-55}$-induced EAE on day 15 , i.e., at the time of the EAE peak. The treatment reduced the severity of EAE in the chronic phase, and the treated mice were symptom-free at the end of the observation period (30 d.p.i.). Importantly, Treg-treated mice were reimmunized with $\mathrm{MOG}_{35-55}$ at 30 d.p.i., and only one mouse developed any clinical manifestations of EAE. This effect on the clinical score occurred in parallel with inhibition of IL-12 and IFN- $\gamma$ expression in the CNS. Interestingly, Tregs were applied to mice intranasally (i.n.), and the authors were able to demonstrate their migratory capacity (presumably via olfactory pathways), as they detected the Tregs within the CNS.

Niedbala et al. used nitric oxide (NO)-induced Tregs in EAE. ${ }^{103}$ They applied Tregs in C57BL/6 mice with MOG $_{35-55}$-induced EAE at day 10, i.e., when the initial clinical signs appeared. The treatment led to a reduction in the severity of chronic EAE throughout the observation period, which ended at 30 d.p.i. This effect occurred in parallel with the limitation of immune cell infiltration into the CNS and a specific reduction in Th17 cell numbers within the CNS.

Malviya et al. generated transgenic $T$ cells expressing a TCR specific for MOG and neurofilament medium (NF-MT) and used these $T$ cells to treat $\mathrm{C} 57 \mathrm{BL} / 6$ mice with EAE induced by $\mathrm{MOG}_{35-55}$ or $\mathrm{PLP}_{178-191 .}{ }^{104}$ These engineered Tregs reduced the severity of EAE when applied at 9 d.p.i. (when clinical symptoms were evident), and their effect increased towards the peak of EAE. Importantly, these Tregs containing TCRs specific for MOG were equally efficient in EAE induced by $\mathrm{MOG}_{35-55}$ and $E A E$ induced by an unrelated CNS antigen, PLP $_{178-191}$. Such efficacy in restricting autoimmune reactivity against unrelated CNS antigens, if extrapolated to humans, would be a beneficial therapeutic property in MS. The engineered Tregs were detected in the CNS of the treated mice, and the authors suggested that the therapeutic effect of the Tregs was achieved within the CNS.

To summarize, at least in the EAE model of MS, a single application of Tregs to mice when the disease is well established is successful and has persistent effects. The cells can be applied systemically (i.v. or i.p.) ${ }^{101,103-111}$ or locally (i.n.). ${ }^{102}$ Nevertheless, the number of cells used for i.n. application was 10 times lower than that used for systemic application, which could be of interest when thinking about translation to humans. However, this difference in the number of Tregs required for efficient application could also be a consequence of different backgrounds and preparations. In conclusion, the results obtained in EAE clearly imply that the application of Tregs is a promising approach for the treatment of ongoing CNS autoimmunity, as observed in MS; therefore, clinical trials with Tregs in MS are expected to yield promising results.

Clinical trials in MS patients. Tregs, mainly tTregs, have been extensively tested in clinical trials for the treatment of not only $A D$ and graft-versus-host disease after bone marrow transplantation as a prophylaxis for solid organ rejection but also for unexpected indications such as thalassemia, muscle dystrophies or amyotrophic lateral sclerosis. ${ }^{92}$ We recently accomplished a phase I/lla trial of autologous $\mathrm{CD}^{+}{ }^{+} \mathrm{CD} 25^{\text {hi }} \mathrm{CD} 127-\mathrm{FoxP}^{+}$Tregs administered to RRMS patients, either i.v. or intrathecally (i.t.) (trial registration: EudraCT: $2014-004320-22 .^{94}$ The therapy proved to be safe. Although very preliminary, the results also suggested that intrathecal administration was more effective than intravenous administration. Experiments with adoptive transfer of Tregs suggested the good safety profile of Tregs administered via both tested routes. 94

Dose, timing, and route of administration. To translate a treatment from early animal use into late human pathology, the dose and route of administration of the cells should be adjusted to 
address the level of inflammation and follow the progression of the disease. We tried to address these issues with the use of two routes of Treg administration. Patients treated i.v. received $40 \times$ $10^{6}$ Tregs/kg b.w., which in our experience is a relatively high dose. Within this arm of the trial, we tried to address the hypothesis that systemic dysregulation between Tconvs and Tregs triggers the disease and relapse. ${ }^{112}$ The results suggest that we were, at least partially, 'too late', as half of the treated patients experienced relapse and progression of disease, as confirmed with MRI. This somewhat confirms that the clinical onset of disease may occur very late in the pathogenesis of MS, when the core of the process has already moved from the periphery to the CNS. The initiation of RRMS occurs somewhere in the peripheral lymphoid system with the presentation of myelin peptides and the generation of autoaggressive Tconvs, as in EAE, which is not adequately controlled by Tregs. ${ }^{113}$ However, Tconvs very quickly traffic to the CNS, destroy the BBB, attack myelin sheaths, and cause the development of lesions. Hence, the systemic administration of drugs is of limited value when symptoms have already occurred. The results of immunophenotyping in our trial seem to confirm such overactivity of Tconvs, which were mainly of an 'experienced' memory phenotype in all patients. At the same time, the majority of Tregs were naïve, confirming their relative inactivity. Surprisingly, MS patients exhibited an extraordinarily high percentage of peripheral Helios ${ }^{-}$FoxP $^{+}$Tregs $(20-30 \%$ of all FoxP3 $^{+}$Tregs). These cells arise during the immune response, which suggests a history of long/massive immune activation in the periphery, with ineffective regulation of this process in MS. There have been reports that Tregs in RRMS patients follow autoreactive Tconvs and, attracted by inflammation, move quickly to the CNS, accumulating in the CSF. Moreover, remission occurs only when Tregs have accumulated in the CSF. ${ }^{114}$

This finding justifies the second arm of our trial, in which patients received Tregs i.t. The dose could be lower $\left(1.0 \times 10^{6}\right.$ Tregs) in these patients, as $100 \%$ of the cells were delivered behind the BBB. The patients did not experience relapse, and MRI confirmed stable nonprogressing lesions in the CNS of these patients, which proved that this approach should be further tested in future trials. ${ }^{94}$

Novel Treg therapies. The approach based on engineered Tregs came from cancer studies in which the receptor specific for a particular molecule expressed on cancer cells was inserted into Tconvs using a vector (chimeric antigen receptor T (CAR-T) cells). This approach allows CAR-T cells to identify and kill cancer cells in a very specific and efficient way. Several drugs based on this therapy, i.e., axicabtagene ciloleucel (Yescarta, Gilead) and tisagenlecleucel (Kymriah, Novartis), are already routinely used. The first CAR-Tregs were constructed with specificity towards alloHLA to quench the possible rejection of an allotransplant. ${ }^{112,115,116}$ The challenge is much higher in ADs such as MS, in which the target antigens are not as obvious. In the majority of such diseases, the complete list of autoantigens is not known. ${ }^{117}$ Moreover, it is possible that the target antigens evolve with the progression of the disease due to epitope spread. ${ }^{114}$ The complexity of the response is also caused by the fact that the same epitopes can trigger responses in both Tregs and Tconvs, and the final outcome depends on which subset prevails. ${ }^{118}$ Nevertheless, there have been attempts to create Tregs with engineered TCRs to direct them to particular sites and protect particular organs from autoimmune attack. ${ }^{119}$ These attempts for MS are at the EAE stage. There have been reports on CAR-T cells with specificity towards MOG manufactured from Tconvs and directed toward a regulatory function through foxP3 gene delivery. This cellular product trafficked to the brain and exerted suppressive activity. ${ }^{102}$ More recently, human Tregs that had a transgenic TCR specific for MBP and had proven immunosuppressive activity were described. ${ }^{111}$
Mesenchymal stem cells

MSCs are nonhematopoietic multipotent and self-renewing progenitor cells with the potential to differentiate into different lineages under specific conditions. They were described for the first time in 1968 by Friedenstein et al. as an adherent fibroblastlike population in the bone marrow that was able to differentiate into adipocytes, chondrocytes and osteocytes. ${ }^{120}$ In 1991, Caplan et al. named these cells "mesenchymal stem cells". ${ }^{121}$ The authors demonstrated that MSCs are involved in bone and cartilage turnover and examined how surrounding conditions play a crucial role in their differentiation. Therefore, MSCs were postulated to be a novel therapeutic strategy for self-cell repair. ${ }^{121}$

MSCs can be isolated from various tissues, including the bone marrow, adipose tissue, placenta, umbilical cord, fetal liver, muscle, and lung. Among these tissues, bone marrow and adipose tissue are the most widely used sources of MSCs for therapeutic purposes. According to the Mesenchymal and Tissue Stem Cell Committee of the International Society for Cellular Therapies, $^{122}$ the cells must be plastic adherent and able to differentiate into osteoblasts, adipocytes and chondroblasts, and their phenotype must be characterized by flow cytometry as $\mathrm{CD}_{105}{ }^{+} \mathrm{CD}{ }^{+} \mathrm{CD}^{+} 0^{+} \mathrm{CD}^{-} 5^{-} \mathrm{CD}_{3}{ }^{-} \mathrm{CD}^{-} 4^{-} / \mathrm{CD} 11 \mathrm{~b}^{-} \mathrm{CD}^{-} \mathrm{Ca}^{-} /$ CD19- HLA-class II $^{-}$. MSCs can be easily cultured and expanded ex vivo and have several properties, such as functions in tissue repair and homeostasis maintenance, immunomodulatory properties and low immunogenicity.

Due to their potential as an immunomodulatory and regenerative therapy, MSCs have been considered an optimal candidate cellular therapy for inflammatory and neurodegenerative diseases of the CNS, such as MS.

Preclinical studies. MSC-based cell therapy is the most investigated and has been examined in numerous in vitro and in vivo studies (EAE). In vivo treatment of EAE mice with bone marrowderived MSCs (BM-MSCs) using several routes of administration (i.v., intraventricular (i.v.t.), and i.p.) has shown clinical amelioration of EAE severity, with reduced inflammatory infiltration, demyelination, and axonal damage. Of note, this beneficial effect was not found when BM-MSCs were infused during the chronic phase of the disease. ${ }^{123}$

It has been widely reported that MSC therapy in EAE exerts an important immunomodulatory effect and, to a lesser extent, a neuroprotective effect that results in axonal and neuronal protection through the release of antiapoptotic, antioxidant, and neurotrophic factors (systematically reviewed in ${ }^{124}$ ). Induction of Tregs, TGF- $\beta 1$, and IL-10 mRNA in the spleen and lymph nodes of treated mice was the main immunological mechanism involved in the induction of peripheral tolerance following MSC transplantation in EAE mice.

Mechanisms of action of MSCs. The specific mechanisms that mediate the clinical benefits of BM-MSCs likely involve a combination of peripheral autoimmune modulation and the induction of CNS tissue protection. MSCs have four main properties: (1) migration capacity, (2) immunomodulation, (3) differentiation and neuroregeneration, and (4) secretion of soluble factors.

After their systemic administration, MSCs can migrate to and engraft in inflamed locations, exerting a local effect. Injured cells and immune cells involved in the immune response regulate MSC migration through the secretion of a broad range of signals, such as growth factors and chemokines. In vitro studies proved that MSC migration is regulated by receptors such as platelet-derived growth factor and insulin-like growth factor 1 and chemokine receptors such as CCR2, CCR3, CCR4, and CCL5. ${ }^{125}$ Studies in animal models have shown that MSCs can roll and tether to the endothelium, crossing the BBB through the VLA-4/VCAM-1 interaction. Moreover, MMPs play an important role in the transit 
of MSCs through the endothelial membrane. Interestingly, it is important to keep in mind that culture conditions during MSC ex vivo expansion can affect the expression of some receptors, such as VLA-4 and MMP, thus altering the migration capacity of MSCs. ${ }^{125-127}$

The immunomodulatory properties of MSCs can be exerted via cell-to-cell interactions or paracrine effects. On T cells, MSCs inhibit $\mathrm{T}$ cell proliferation via a mechanism independent of apoptosis induction. ${ }^{128}$ After coculture with T cells, MSCs decreased the Th1 response and induced a switch towards the Th2 response (a decrease in IFN- $\gamma$ secretion and an increase in IL-4 secretion). ${ }^{129}$ In addition, MSCs induce the expansion of Treg subsets, increasing Foxp3 and CD25 expression. These Treg cells express TGF- $\beta 1$ and PGE2. ${ }^{129,130}$

Regarding B cells, it has been reported that PDL1 inhibits their proliferation in murine cells and arrests the cell cycle in human cells. ${ }^{131,132}$ Chemotactic properties are also affected by soluble factors secreted by MSCs. A decrease in the expression of some chemokine receptors, such as CXCR4, CXCR5, and CCR7, was observed in B cells, together with a decrease in CXCL12 (a CXCR4 ligand) and CXCL13 (a CXCR5 ligand) expression in MSCs. In contrast, B cell costimulatory molecule expression and cytokine production were unaltered. ${ }^{132}$ Furthermore, suppression of B cell terminal differentiation by soluble factors secreted by MSCs, such as MCP-1 or IL-6, was also reported in C57BL/6 mice. ${ }^{133}$

MSCs induced the inhibition of NK cell proliferation when cocultured in IL-15-supplemented medium via both cell-to-cell contact and soluble factors, such as TGF- $\beta 1$ and PGE2. Moreover, under these conditions, a decrease in the production of IFN- $\gamma$ and IL-10, a decrease in the surface expression of CD56 in NK cells (although no changes were observed in the ratio of the CD56 dim and CD56 $6^{\text {bright }}$ subsets), and lower cytotoxicity against HLA class I targets were found. ${ }^{12,134,135}$

MSCs can inhibit the maturation of DCs, resulting in a decrease in their capacity to activate alloreactive T cells. Furthermore, MSCs can inhibit TFN-a release by DCs, resulting in a tolerogenic state. Moreover, it has been postulated that PGE2 secreted by MSCs plays an important role in promoting the Th2 cell response, acting against Th1 cells, during the DC-induced Th cell response. ${ }^{129,136}$

As previously mentioned, MSCs release multiple soluble and encapsulated molecules, such as growth factors, cytokines, and chemokines (secretome), which can exert a biological effect in tissues. The conditioned medium of MSCs was shown to have a beneficial effect in damaged tissue in the liver and myocardium. Among these molecules are PGE2, which has anti-inflammatory and antiproliferative effects; IL-10 and IL-1 receptor antagonist (which have anti-inflammatory effects); and TGF- $\beta 1$ and hepatocyte growth factor (which suppress T cell proliferation). ${ }^{126,127}$ Moreover, it was recently reported that exosomes released by MSCs can cross the BBB due to their small size and transfer bioactive molecules. In this regard, MSC-derived exosomes polarized microglial cells mainly into the $M 2$ anti-inflammatory phenotype, promoting a shift to an antiinflammatory profile and, consequently, reducing the clinical symptoms of EAE in rats. ${ }^{137}$

One important characteristic of MSCs is their ability to differentiate towards both mesenchymal and ectodermal cells, such as neurons, astrocytes, and oligodendrocytes. When analyzing the neuroprotective and regenerative functions of MSCs in MS, different experiments demonstrated that after i.v. administration of MSCs, they spread and homed preferentially to inflamed tissues, to the CNS in the case of EAE, to induce neuronal axon protection and the regeneration of damaged areas. ${ }^{138,139}$ Unfortunately, MSCs have not been demonstrated to transdifferentiate into neuronal cells. Their protective function is likely related to the secretion of antiapoptotic, anti-inflammatory, and neurotropic factors (through the activation of astroglial cells to secrete neurotrophins such as BDNF, glial cellderived neurotrophic factor, and nerve growth factor), ${ }^{139}$ as well as the probable recruitment of local progenitor cells for subsequent differentiation into neurons and oligodendrocytes. ${ }^{139-141}$ These promising results encourage the use of MSCs in MS.

Clinical trials in MS. The number of clinical trials investigating MSC treatment for RRMS and progressive MS patients has increased quickly over the past two decades. Currently, more than twenty clinical trials are registered at ClinicalTrials.gov (see Table 4). Thus far, the results have shown that MSC therapy in MS patients is safe, with no relevant side effects. ${ }^{142-151}$ Due to variability in the protocols used (different types of MSCs, routes of administration, and doses) and the limited number of patients enrolled in each trial, most studies have been unable to draw conclusions about the efficacy of these treatments. Nevertheless, some trials have reported beneficial effects of MSCs through a decrease in relapse rate or disability. ${ }^{143,146,147}$

To establish a consensus protocol for the use of MSCs for treating MS patients, in 2010, a group of experts created the International Mesenchymal Stem Cell Transplantation Study Group (IMSCTSG). As a result, a large multicenter randomized, doubleblind, crossover phase I/II clinical trial was initiated to analyze the safety and efficacy of a single i.v. dose of autologous BM-derived MSCs (Mesenchymal StEm cells for Multiple Sclerosis: MESEMS trial; NCT02403947). ${ }^{142}$ The results of this trial have not yet been published.

Recently, Sarkar et al. reported relevant data about the MSC secretome that must be considered in the context of treatment with MSCs. These authors found that chronic inflammatory stress in MS patients limits MSC functionality by altering the MSC secretome. ${ }^{152}$ Moreover, other factors, such as aging and the in vitro expansion of MSCs, also exert detrimental effects on their functionality. ${ }^{152}$ Therefore, an in-depth analysis of the autologous MSC secretome should be considered as a quality control measure before administrating MSC therapy.

Regarding the effect of MSC treatment on the immune response, only a few clinical trials have performed immune monitoring to elucidate the mechanism of action of this therapy. Llufriu et al. reported a decrease (although it was not statistically significant) in Th1 and Th17 cells and an increase in Breg cells, as analyzed by the expression of IFN- $\gamma$, IL-17, and IL-10, respectively, using intracellular cytokine staining. ${ }^{143}$ Karussis et al. described an increase in Treg cells (identified as $\mathrm{CD} 4^{+} \mathrm{CD} 25^{+}$), together with a decrease in the expression of CD83 and CD86 in DCs and CD40 in activated cells. Moreover, they performed functional analysis of the $\mathrm{T}$ cell response, which showed a decrease in proliferation in response to phytohemagglutinin (PHA). ${ }^{147}$ Finally, a clinical trial of the administration of autologous BM-MSCs is currently ongoing in Jordan and will analyze the levels of $\operatorname{lgG}, \lg A$, and $\lg M$ and complement factors C3 and C4 in treated patients (NCT03069170).

\section{Hematopoietic stem-cell transplantation}

Hematopoietic stem-cell transplantation (HSCT) was established for the treatment of hematological malignancies such as multiple myeloma and leukemias due to the capacity of hematopoietic stem cells to differentiate into all hematopoietic cell types. ${ }^{153}$ Surprisingly, treated patients who had concomitant ADs experienced amelioration of their clinical symptoms following HSCT. ${ }^{154}$ As a result, high-dose immunosuppression followed by autologous HSCT (aHSCT) has been investigated for patients with severe MS, with the rationale of this therapy based on a "reset" of the immune repertoire to eliminate autoreactive $T$ and $B$ cells; thus, subsequent aHSCT would allow reconstitution with the hope that a new and more self-tolerant immune system is developed. ${ }^{55}$

HSCT is carried out through different steps: mobilization, harvesting, ablative conditioning, and transplantation of aHSCs. ${ }^{156}$ First, HSCs are collected from the peripheral blood of patients after the mobilization of HSCs from bone marrow using treatment with granulocyte colony-stimulating factor (G-CSF) or GM-CSF with cyclophosphamide to prevent possible MS relapse or worsening of 


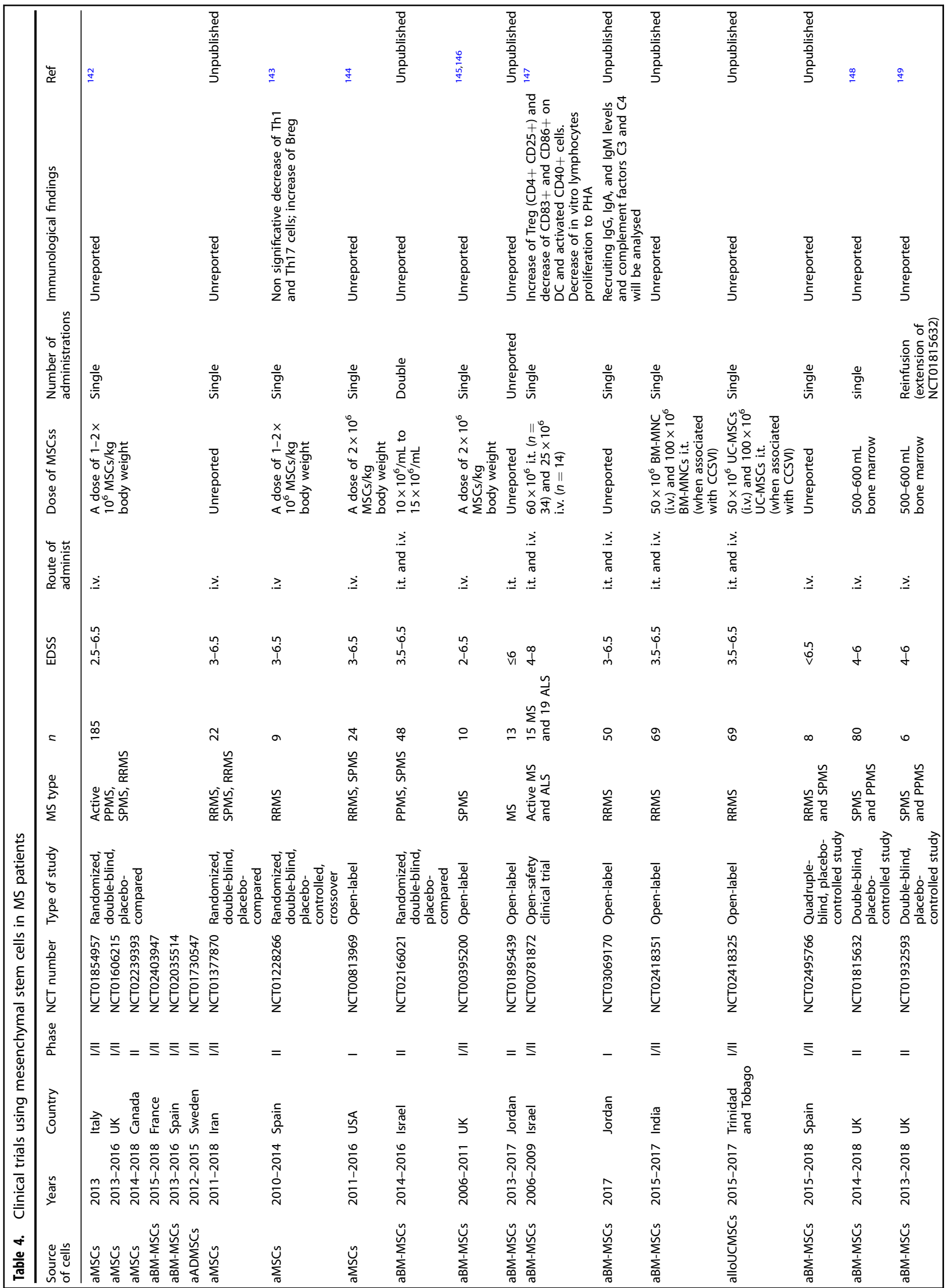




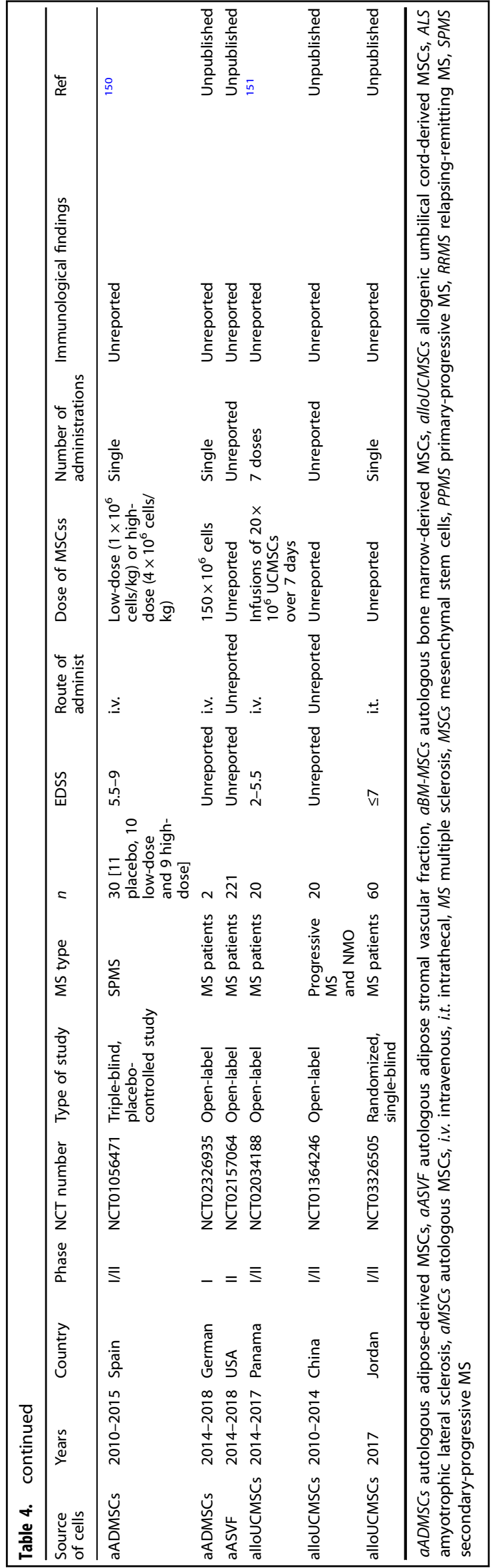

clinical symptoms as a result of G-CSF or GM-CSF administration. ${ }^{156}$ After 4 or 5 days, cells are harvested by leukapheresis and cryopreserved. Additionally, an HSC purification step is performed by CD34 positive selection to eliminate possible autoreactive lymphocytes. Then, the patient receives ablative conditioning to eradicate autoreactive cells. Different regimens of immune ablative conditioning are used based on the intensity of ablation using different chemotherapeutics and immunosuppressive drugs. High-intensity ablative regimens involve a high i.v. dose of immunosuppressive therapy and are associated with high toxicity and, in a small percentage of patients, mortality. ${ }^{156,157}$ In contrast, low-intensity regimens are nonmyeloablative and produce fewer adverse effects but may be associated with the early reappearance of MS disease activity post infusion. Hence, intermediate-intensity ablative regimens, referred to as BEAM (BCNU (or carmustine), etoposide, cytosine arabinoside, melphalan) or modified BEAM, are becoming more accepted. ${ }^{157}$ Following ablative conditioning, cryopreserved HSCs are thawed and reinfused into the patient.

Despite the promising positive results obtained using aHSCT (see below), some aHSCT-related risks must be considered: as stated above, the risk of transplant-related mortality, which is most severe in the first 100 days after transplantation, and the increased susceptibility to infection as a result of the accompanying chemotherapeutic immunosuppressive regimen. In addition, long-term side effects include the development of secondary autoimmune problems and/or fertility issues. ${ }^{158}$ Interestingly, aHSCT has dramatically improved over the years, showing a $0.3 \%$ treatment-related mortality rate since $2005 .^{157}$

Clinical trials in MS. Since the end of the 1990s, several clinical trials evaluating the safety and efficacy of aHSCT using different conditioning regimens have been performed. Published results have shown that aHSCT can inhibit MS disease activity for 4-5 years in $70-80 \%$ of patients. Interestingly, this rate is higher than that achieved with any other therapy for MS. The results were better in young patients with inflammatory-active RRMS (reviewed in ${ }^{157}$ ).

To go one step further in examining aHSCT, currently, a phase III randomized clinical trial in RRMS patients with significant inflammatory disease activity is being conducted to compare the efficacy of aHSCT using a nonablative conditioning regimen with that of alemtuzumab (anti-CD52), which is considered the most effective available drug for RRMS (NCT03477500). Importantly, if the results indicate improved efficacy of aHSCT over alemtuzumab, aHSCT will likely be approved as a part of the current standard treatment recommendations for a significant proportion of RRMS patients (except in the case of Sweden, where aHSCT has been already approved). In the same way, another phase III clinical trial to analyze the efficacy of aHSCT (using high-dose myeloablative conditioning) in comparison with the best available therapy (BAT) in treatment-resistant RRMS is currently ongoing (BEAT-MS trial, NCT04047628). This multicenter, randomized, blinded study was conducted in a total of 156 RRMS patients distributed to each treatment arm at a ratio of 1:1. Treatments included in the BAT arm are natalizumab (anti-CD49d), alemtuzumab (anti-CD52), ocrelizumab (antiCD20) and rituximab (anti-CD20). Relapse-free survival up to 3 years will be determined and used to compare the efficacy of aHSCT with that of the other treatments.

T cell vaccination

Autologous $\mathrm{T}$ cell vaccination (TCV) involves collecting and expanding myelin-reactive T cells from MS patients and reinfusing them after their attenuation by irradiation. The rationale is that, as a result of this process, the immune system will attack pathogenic myelin-specific T cells, causing their deletion or inactivation while maintaining protective immunity. 
The first study using attenuated MBP-reactive T lymphocytes was conducted in Lewis rats in $1981 .^{159}$ The adoptive transfer of MBP-reactive $T$ cells in rats induced EAE disease onset. Interestingly, administration of the same MBP-T cells attenuated by irradiation before adoptive induction resulted in disease prevention. Increased interest in TCV led to the initiation of multiple trials in MS patients in the late 1990s. The results from phase I pilot studies demonstrated that TCV treatment was safe and well tolerated $^{160-165}$ (Table 5) and depleted MBP-reactive T cells after only 2 administrations. ${ }^{160}$ Interestingly, a correlation between MBP-reactive T cell depletion and a $40 \%$ reduction in the relapse rate was found in RRMS patients. Nevertheless, no relevant reduction in EDSS score was observed in the RRMS patients. In contrast, a slight increase in EDSS score after 2 years was reported in SPMS patients ${ }^{166}$ (Table 5).

To improve TCV outcomes in MS patients, T lymphocytes isolated from CSF were used to develop CSF-derived $T$ cell lines against myelin peptides, since CSF was thought to contain infiltrating pathogenic lymphocytes relevant to the disease process due to the proximity of these lymphocytes to the CNS. Data from two pilot studies indicated that the administration of attenuated autoreactive CSF-derived T cell clones was feasible and safe, and no adverse effects were observed. Phase II studies with a large number of MS patients are required to evaluate the clinical and radiological efficacy of CSF-derived TCV. ${ }^{167,168}$

To improve clinical remission, a new strategy using multivalent TCV was developed using immune-dominant epitope sequences of MBP, MOG and PLP. A phase I clinical trial was conducted by Achiron et al. using attenuated T cell lines specific for different MBP and/or MOG peptides. ${ }^{169}$ The results of the trial showed clinical as well as radiological benefits with no adverse effects in RRMS patients who did not respond to disease-modifying treatments. ${ }^{169}$ In addition, multivalent TCV allows personalized therapy following prescreening for myelin reactivity. In this regard, Tcelna (Imilecleucel-T, previously known as Tovaxin), a TCV composed of autologous preselected $T$ cells reactive against up to six immunodominant peptides derived from MBP, MOG and PLP, was shown to be safe and well tolerated in a phase II clinical trial; however, its clinical and radiological efficacy was not demonstrated (TERMS trial: Tovaxin for Early Relapsing Multiple Sclerosis, NCT00245622, ${ }^{170}$ ). An extension study (OLTERMS trial: Open label extension of TERMS study, NCT00595920) was initiated to further evaluate the clinical efficacy of Tcelna/ Tovaxin. However, the study was terminated because it did not accomplish the predefined primary endpoint of reducing brain atrophy or the secondary endpoint of decreasing disability progression (NCT00595920).

\section{COMBINED THERAPIES}

Evidence from phase I clinical trials conducted in type 1 diabetes, rheumatoid arthritis, Crohn's disease and MS patients has demonstrated that tolDCs, Tregs, TCV, MSCs, and HSCT are safe and well tolerated. However, due to the different cell types and mechanisms involved in the maintenance of immune tolerance and the difficulty of establishing an optimal dose, route, and frequency of administration, it has been postulated that a combined therapy of antigen-specific cells with conventional immunomodulatory drugs is most likely necessary to potentiate their beneficial effects and restore immune homeostasis. ${ }^{54}$

TolDCs with immunomodulatory/immunosuppressive drugs A single infusion of donor-derived VitD3+IL-10-tolDCs before transplantation in combination with $\mathrm{CD} 28$ costimulatory signal blockade using the fusion protein CTLA-4 lg (abatacept) and rapamycin significantly prolonged allograft survival in nonhuman primates by attenuating donor-reactive memory T cells. ${ }^{171}$

More recently, the results of the ONE Study, an analysis of seven non-randomized, single-arm, phase $\mathrm{I} / \mathrm{lla}$ trials in living donor kidney transplantation in which different types of regulatory cells, including tolDCs, were combined with conventional immunosuppressive drugs reducing the proliferative response of lymphocytes by inhibiting IL-2 signaling (basiliximab and tacrolimus), blocking de novo synthesis of guanosine nucleotides (mycophenolate mofetil) or using a feedback mechanism to control inflammation and the immune response to steroids. Data from the trial showed the safety and feasibility of combined therapy. Interestingly, a decrease in infectious complications was reported in the group administered combined therapy. ${ }^{100}$ In this context and to move one step forward in the clinical application of tolDCs in MS, our group investigated the effect of tolDCs combined with IFN- $\beta$. We found that the anti-inflammatory and immunomodulatory properties of IFN- $\beta$ combined with VitD3-tolDCs induced a reduction in Th1 and Th17 cells, favoring a more potent antigen-specific regulatory effect of VitD3-tolDCs both in vivo (EAE model) and in vitro in cultures of peripheral blood cells from MS patients (Quirant-Sánchez et al. unpublished data).

Tregs with immunomodulatory/immunosuppressive drugs The relationship between Tregs and IL-2 is probably the most explored area in MS research, with the longest history of clinical trials. ${ }^{172}$ Tregs have high expression of IL-2R and are highly dependent on IL-2; therefore, this cytokine or its muteins have been used alone or in combination with Tregs in many conditions, such as GvHD, transplantation or T1D. ${ }^{96,97}$ Other approaches include the administration of rapamycin as an immunosuppressive agent able to induce Tregs and tolDCs ${ }^{98,99}$ or adjuvant therapy with Tregs added to standard immunosuppression in organ transplantation. ${ }^{100}$ Our team has been testing Tregs combined with anti-CD20 antibody in T1D. ${ }^{173}$

TolDCs in combination with Tregs

The combination of toIDC and Treg cell therapy has been proposed for the treatment of ADs since these cells can interact to stabilize, maintain and potentiate their tolerogenic effects. ${ }^{174}$ Although coadministration or serial administration of antigenspecific tolDCs and Tregs a priori does not seem to be feasible, a single leukapheresis could provide enough monocytes to generate tolDCs and lymphocytes for autologous Treg expansion. ${ }^{174}$ However, to date, no studies investigating the efficacy of this combined therapy have been reported.

\section{TolDCs/Tregs in combination with MSCs}

A different combination therapy approach for ADs is the administration of antigen-specific cells, such as tolDCs or Tregs, with MSCs. In this context, synergistic suppression of autoimmune arthritis was reported when collagen-induced arthritis (CIA) mice were treated with RelB-silenced tolDCs and MSCs. Combined therapy was able to inhibit disease progression, decrease the clinical symptoms of $\mathrm{CIA}$ and reduce joint damage. Immunological studies revealed inhibition of the collagen $T$ cell response and $a$ shift towards an anti-inflammatory profile, although the most potent synergistic effect elicited by RelB-silenced tolDC and MSC therapy was a strong reduction in Th17 cells. ${ }^{175}$

Another example was published by Lee et al., who used a murine model of acute GVD (aGVHD) to determine the efficacy of combined Treg and MSC therapy. ${ }^{176}$ Researchers have shown that the adoptive transfer of donor-derived MSCs and Tregs reduces the severity of aGVHD by controlling Th1 and Th17 responses (related to the function of Tregs), accompanied by increased long-term survival of transferred Tregs and induction of endogenous Treg repopulation in target organs (related to MSC function). ${ }^{176}$

In conclusion, all these data suggest that combination therapies have the advantage of increasing the possible clinical effectiveness of antigen-specific cell-based tolerogenic therapies and will contribute to their optimal application in the future. 


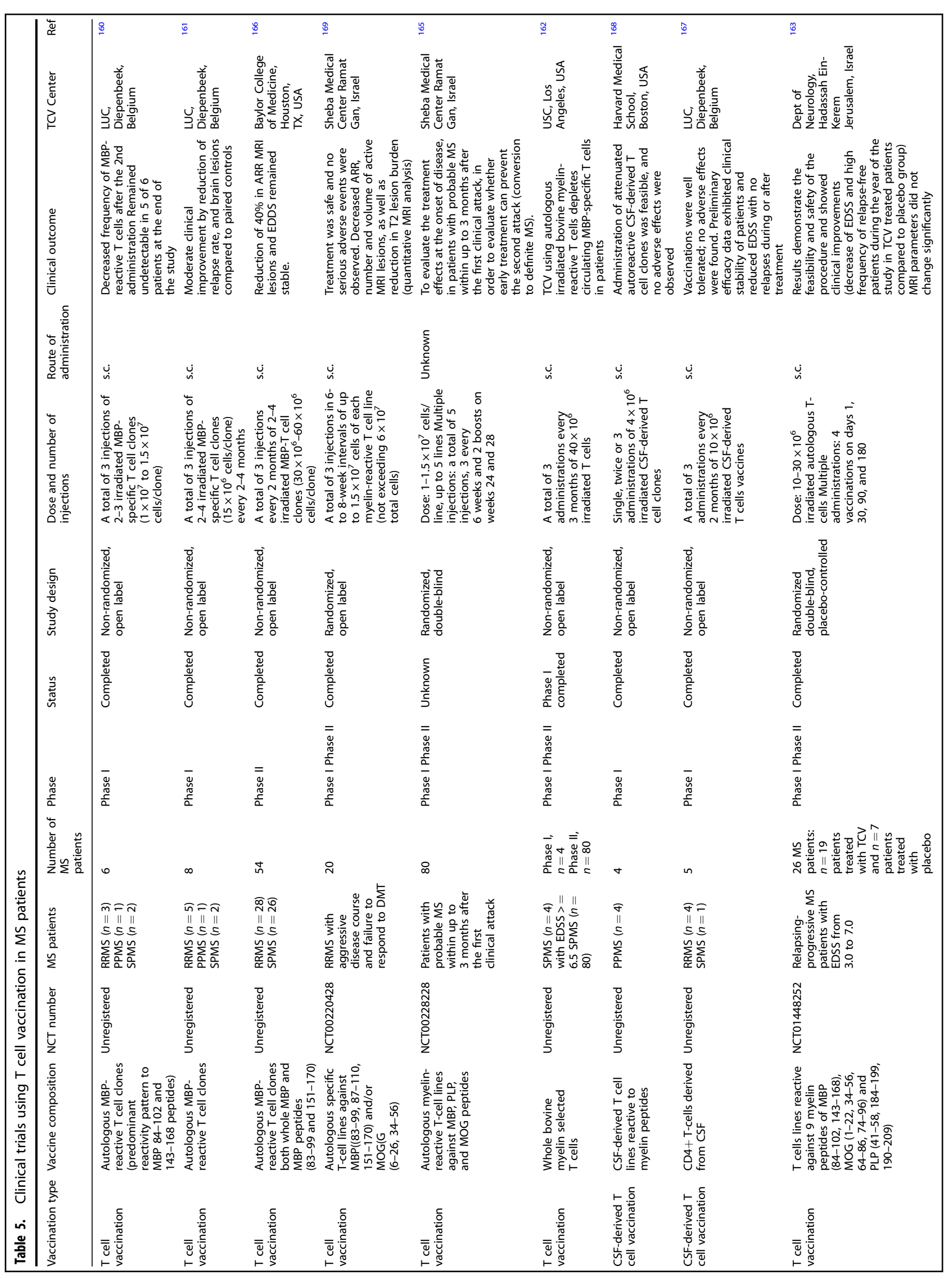




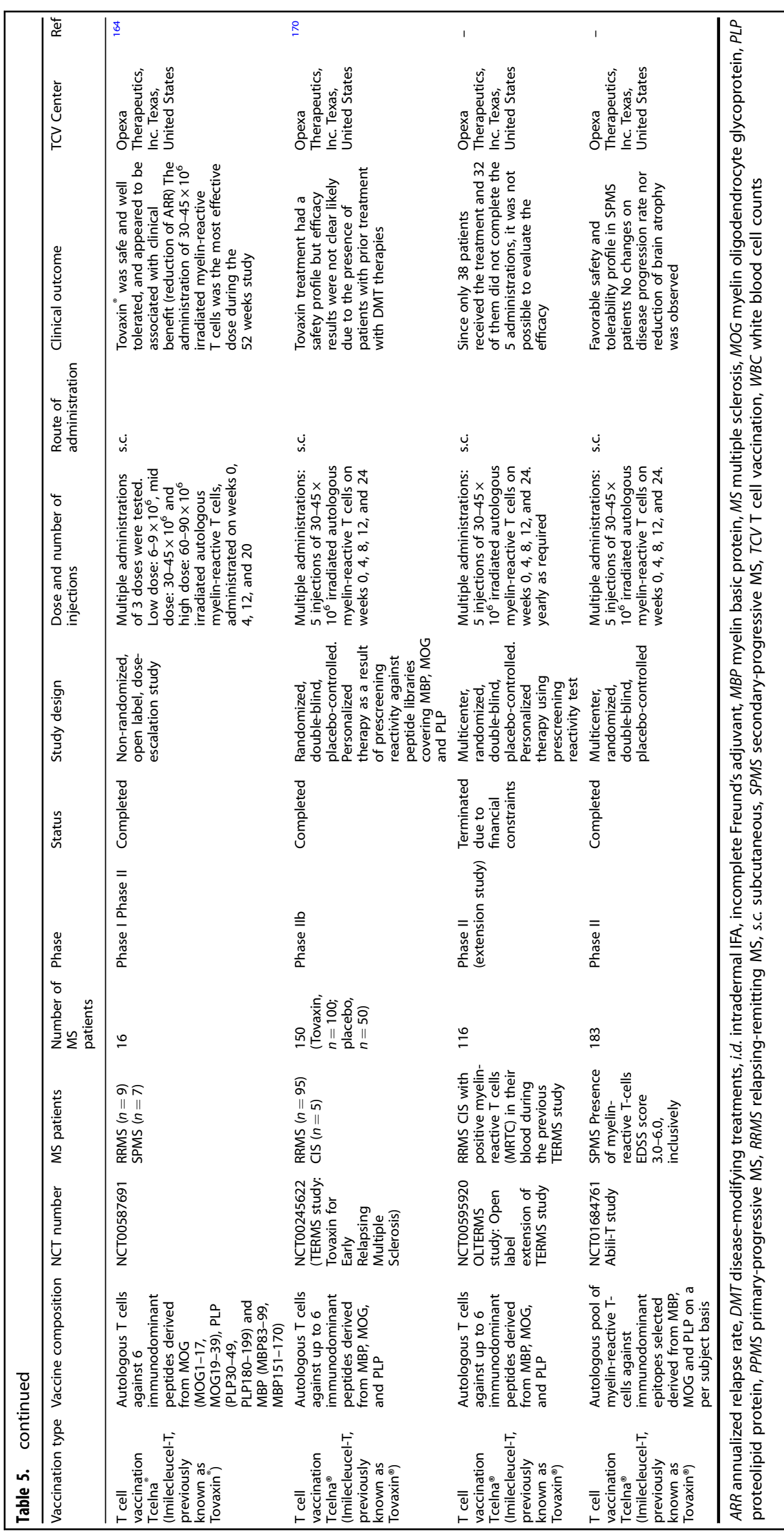




\section{LEGAL RULES FOR CELLULAR PRODUCTS}

Cellular therapies are classified as either transplants, in which unmodified cells are immediately administered, or drugs, in which substantial laboratory modification of the cells and/or nonhomologous use of the cells occurs. In Europe, the use of these two kinds of products is regulated by directive 2004/23/EC, which sets standards of quality and safety for the donation, procurement, testing, processing, preservation, storage and distribution of human tissues and cells, and directive 1394/2007, which is focused on advanced therapy medicinal products (ATMPs). Clinical trials to test new investigational medicines are also centrally regulated by regulation 536/2014. In general, these directives implement the guidelines established by the International Council for Harmonization of Technical Requirements for Pharmaceuticals for Human Use (ICH), a common worldwide effort of national regulatory authorities and the pharmaceutical industry to discuss scientific and technical aspects of pharmaceuticals. Similar acts based on $\mathrm{ICH}$ rules are also in place in other parts of the world and describe the path of new medicines from discovery to routine use. This path for cellular drugs is similar to that for other drugs. It starts from preclinical assessment, which establishes the most important toxic, pharmacokinetic and pharmacokinetic features of the investigational drug. This step in humans consists of three trials: a first-in-man phase I trial to establish safety and dose, a phase II trial to test efficacy and a phase III trial to confirm safety and efficacy in a larger population. The last study is usually pivotal and the basis for marketing authorization. Drugs are also continuously surveyed post authorization, which is phase IV. In Europe, the authorization of cellular drugs is centralized, as it occurs in one step for all EU countries and is granted by the EMA. Some possibilities for early access to cellular products, such as hospital exemption rules, exist, but these are very limited, and there is pressure to provide standardized, equal treatment to all citizens of the EU. The importance of cellular drugs and high expectations that these cellular drugs will be a 'game changer' for many unmet medical needs are highlighted by the fact that there is a dedicated legal board within the EMA, the Committee for Advanced Therapies (CAT), which addresses only regulations on cellular therapy. There are also academic initiatives to regulate the way cellular drugs are manufactured. For example, academic guidelines for tolerance-inducing cellular products based on the minimal information model exist. ${ }^{177,178}$

\section{CONCLUDING REMARKS}

Over the past 20 years, an extraordinary effort has been made to develop treatments that can halt the natural evolution of MS. Thanks to this effort, we have a variety of drugs that, due to their powerful anti-inflammatory/immunosuppressive effects, decrease the rate of relapse and radiological activity, thus slowing the onset of disability. However, by acting in an immunologically nonspecific manner and suppressing one or more branches of the immune response, these treatments have the potential to cause serious adverse effects. In this context, cell therapy appears to be a promising strategy.

Phase I clinical trials with tolDCs, MSCs and Tregs in MS patients have shown these therapies to be safe and well tolerated, with no relevant adverse effects. Among cell-based strategies, MSCs have a potent immunomodulatory effect. TolDCs loaded with selfantigens against which tolerance is induced, Tregs, and more recently CAR-Tregs have the potential to specifically act against the cause of the disease, i.e., the autoimmune response to CNS myelin, while maintaining protective immunity. In addition, although less explored, some cell therapies show neuroprotective and neurorepair potential. These cells have shown promising results in experimental models, and some, such as hfNSCs, MSCNPs and hESCs, are already being tested in patients.

All these developments paint a very hopeful picture for the next few years. The personalized combination of treatments will allow us to approach this disease from various fronts and, without a doubt, increase the possibility of obtaining a suitable therapy for each patient at the right time to ensure the highest quality of life possible because each patient is unique, as is their disease.

\section{ACKNOWLEDGEMENTS}

This review was possible thanks to contributions and funding from the European Union's Horizon 2020 research and innovation program under grant agreement 779316 (RESTORE); the support of project PI17/01521 and PI20/01313, integrated in the Plan Nacional de I+D + I and cosupported by the ISCIII-Subdirección General de Evaluación and the Fondo Europeo de Desarrollo Regional (FEDER); and the support of National Centre for Research and Development, Poland (grant $n^{\circ}$ STRATEGMED1/233368/1/NCBR/ 2014). MJ.M. is beneficiary of a Sara Borrell contract from the ISCIII and the FEDER. N.D. and Đ.M. are supported by MPNTR Republika Srbija, grant $n^{\circ} 451-03-68 / 2020-14 / 200007$. F.F. holds a PhD fellowship from the INsTRuCT Consortium focused on developing innovative myeloid regulatory cell (MRC)-based immunotherapies, which receive an Innovative Training Network subsidy from the European Union Horizon 2020 Programme.

\section{ADDITIONAL INFORMATION}

Competing interests: The authors declare no competing interests.

\section{REFERENCES}

1. Lublin, F. D. \& Reingold, S. C. Defining the clinical course of multiple sclerosis: results of an international survey. Neurology 46, 907-911 (1996).

2. Lublin, F. D. et al. Defining the clinical course of multiple sclerosis: the 2013 revisions. Neurology 83, 278-286 (2014).

3. Thompson, A. J. et al. Diagnosis of multiple sclerosis: 2017 revisions of the McDonald criteria. Lancet Neurol. 17, 162-173 (2018).

4. Bielekova, B., Sung, M.-H., Kadom, N., Simon, R., McFarland, H., \& Martin, R. Expansion and functional relevance of high-avidity myelin-specific CD4 $+\mathrm{T}$ cells in multiple sclerosis. J. Immunol. 172, 3893-3904 (2004).

5. Olsson, T., Barcellos, L. F. \& Alfredsson, L. Interactions between genetic, lifestyle and environmental risk factors for multiple sclerosis. Nat. Rev. Neurol. 13, 26-36 (2016).

6. Papenfuss, T. L. et al. Estriol generates tolerogenic dendritic cells in vivo that protect against autoimmunity. J. Immunol. 186, 3346-3355 (2011).

7. Kurth, F. et al. Neuroprotective effects of testosterone treatment in men with multiple sclerosis. Neurolmage Clin. 4, 454-460 (2014).

8. Jelcic, I. et al. Memory B cells activate brain-homing, autoreactive CD4 + T cells in multiple sclerosis. Cell 175, 85-100.e23 (2018).

9. Patsopoulos, N. A. et al. Multiple sclerosis genomic map implicates peripheral immune cells and microglia in susceptibility. Science. 365, eaav7188 (2019).

10. Celarain, N. \& Tomas-Roig, J. Aberrant DNA methylation profile exacerbates inflammation and neurodegeneration in multiple sclerosis patients. J. Neuroinflammation 17, 21 (2020).

11. Haas, J. et al. Reduced suppressive effect of $C D 4+C D 25$ high regulatory $T$ cells on the $\mathrm{T}$ cell immune response against myelin oligodendrocyte glycoprotein in patients with multiple sclerosis. Eur. J. Immunol. 35, 3343-3352 (2005).

12. Nuyts, A. H., Lee, W. P., Bashir-Dar, R., Berneman, Z. N. \& Cools, N. Dendritic cells in multiple sclerosis: key players in the immunopathogenesis, key players for new cellular immunotherapies? Mult. Scler. J. 19, 995-1002 (2013).

13. Dendrou, C. A., Fugger, L. \& Friese, M. A. Immunopathology of multiple sclerosis. Nat. Rev. Immunol. 15, 545-558 (2015).

14. van Langelaar, J., Rijvers, L., Smolders, J. \& van Luijn, M. M. B and T cells driving multiple sclerosis: identity, mechanisms and potential triggers. Front. Immunol. 11, 760 (2020).

15. Rahman, M. T. et al. IFN- $\gamma$ IL-17A, or zonulin rapidly increase the permeability of the blood-brain and small intestinal epithelial barriers: relevance for neuroinflammatory diseases. Biochem Biophys. Res. Commun. 507, 274-279 (2018).

16. Cayrol, R. et al. Activated leukocyte cell adhesion molecule promotes leukocyte trafficking into the central nervous system. Nat. Immunol. 9, 137-145 (2008).

17. Michel, L. et al. Activated leukocyte cell adhesion molecule regulates $B$ lymphocyte migration across central nervous system barriers. Sci. Transl. Med. 11, eaaw0475 (2019).

18. Ransohoff, R. M. \& Engelhardt, B. The anatomical and cellular basis of immune surveillance in the central nervous system. Nat. Rev. Immunol. 12, 623-635 (2012).

19. Louveau, A. et al. Structural and functional features of central nervous system lymphatic vessels. Nature 523, 337-341 (2015).

20. Aspelund, A. et al. A dural lymphatic vascular system that drains brain interstitial fluid and macromolecules. J. Exp. Med. 212, 991-999 (2015). 
21. Lassmann H., Bradl M. Multiple sclerosis: experimental models and reality. Acta Neuropathologica. 133, 223-244 (2017).

22. Robinson, A. P., Harp, C. T., Noronha, A. \& Miller, S. D. The experimental autoimmune encephalomyelitis (EAE) model of MS. utility for understanding disease pathophysiology and treatment. Handb. Clin. Neurol. 2014, 173-189 (2014).

23. Walker-Caulfield, M. E., Hatfield, J. K. \& Brown, M. A. Dynamic changes in meningeal inflammation correspond to clinical exacerbations in a murine model of relapsing-remitting multiple sclerosis. J. Neuroimmunol. 278, 112-122 (2015).

24. Kivisakk, P. et al. Localizing central nervous system immune surveillance: meningeal antigen-presenting cells activate $T$ cells during experimental autoimmune encephalomyelitis. Ann. Neurol. 65, 457-469 (2009).

25. Bartholomäus, I. et al. Effector T cell interactions with meningeal vascular structures in nascent autoimmune CNS lesions. Nature 462, 94-98 (2009).

26. Brown, D. A. \& Sawchenko, P. E. Time course and distribution of inflammatory and neurodegenerative events suggest structural bases for the pathogenesis of experimental autoimmune encephalomyelitis. J. Comp. Neurol. 502, 236-260 (2007).

27. Kammona O., Kiparissides C. Recent advances in antigen-specific immunotherapies for the treatment of multiple sclerosis. Brain Sci. 10, (2020).

28. Baecher-Allan C., Kaskow B. J., Weiner H. L. Multiple sclerosis: mechanisms and immunotherapy. Neuron 97, 742-768 (2018)

29. Abrahamsson, S. V. et al. Non-myeloablative autologous haematopoietic stem cell transplantation expands regulatory cells and depletes IL-17 producing mucosal-associated invariant T cells in multiple sclerosis. Brain 136, 2888-2903 (2013).

30. ISospedra, M. B cells in multiple sclerosis. Curr. Opin. Neurol. 31, 256-262 (2018).

31. Magliozzi, R. et al. Meningeal B-cell follicles in secondary progressive multiple sclerosis associate with early onset of disease and severe cortical pathology. Brain 130, 1089-1104 (2007).

32. Serafini, B., Rosicarelli, B., Magliozzi, R. \& Stigliano, E. A. F. Detection of ectopic Bcell follicles with germinal centers in the meninges of patients with secondary progressive multiple sclerosis. Brain Pathol. 14, 164-174 (2004).

33. Brown, M. A. \& Weinberg, R. B. Mast cells and innate lymphoid cells: underappreciated players in CNS autoimmune demyelinating disease. Front. Immunol. 9, 514 (2018).

34. Greenfield, A. L. \& Hauser, S. L. B-cell therapy for multiple sclerosis: entering an era. Ann. Neurol. 83, 13-26 (2018).

35. Lock, C. et al. Gene-microarray analysis of multiple sclerosis lesions yields new targets validated in autoimmune encephalomyelitis. Nat. Med. 8, 500-508 (2002).

36. Christy, A. L., Walker, M. E., Hessner, M. J. \& Brown, M. A. Mast cell activation and neutrophil recruitment promotes early and robust inflammation in the meninges in EAE. J. Autoimmun. 42, 50-61 (2013).

37. Karussis, D. \& Vaknin-Dembinsky, A. Hematopoietic stem cell transplantation in multiple sclerosis: a review of the clinical experience and a report of an international meeting. Expert Rev. Clin. Immunol. 6, 347-352 (2010).

38. Russi, A. E., Walker-Caulfield, M. E., Guo, Y., Lucchinetti, C. F. \& Brown, M. A. Meningeal mast cell-T cell crosstalk regulates $T$ cell encephalitogenicity. J. Autoimmun. 73, 100-110 (2016).

39. El-Behi, M. et al. The encephalitogenicity of TH 17 cells is dependent on IL-1and IL-23-induced production of the cytokine GM-CSF. Nat. Immunol. 12, 568-575 (2011).

40. Ponomarev, E. D. et al. GM-CSF production by autoreactive T cells is required for the activation of microglial cells and the onset of experimental autoimmune encephalomyelitis. J. Immunol. 178, 39-48 (2007).

41. Croxford, A. L. et al. The cytokine GM-CSF drives the inflammatory signature of CCR2 + monocytes and licenses autoimmunity. Immunity 43, 502-514 (2015).

42. Codarri, L. et al. RORy3t drives production of the cytokine GM-CSF in helper $T$ cells, which is essential for the effector phase of autoimmune neuroinflammation. Nat. Immunol. 12, 560-567 (2011).

43. Rumble, J. M. et al. Neutrophil-related factors as biomarkers in EAE and MS. J. Exp. Med. 212, 23-35 (2015).

44. Pierson, E. R., Wagner, C. A. \& Goverman, J. M. The contribution of neutrophils to CNS autoimmunity. Clin. Immunol. 189, 23-28 (2018)

45. Manda-Handzlik, A. \& Demkow, U. The brain entangled: the contribution of neutrophil extracellular traps to the diseases of the central nervous system. Cells 8, 1477 (2019)

46. Klose, C. S. N. \& Artis, D. Innate lymphoid cells as regulators of immunity, inflammation and tissue homeostasis. Nat. Immunol. 17, 765-774 (2016).

47. Huang, Y. et al. S1P-dependent interorgan trafficking of group 2 innate lymphoid cells supports host defense. Science 359, 114-119 (2018).

48. Weyand, C. M., Kurtin, P. J. \& Goronzy, J. J. Ectopic lymphoid organogenesis: a fast track for autoimmunity. Am. J. Pathol. 159, 787-793 (2001)
49. Gross, C. C. et al. Regulatory functions of natural killer cells in multiple sclerosis. Front. Immunol. 7, 606 (2016).

50. Lünemann, J. D. \& Münz, C. Do natural killer cells accelerate or prevent autoimmunity in multiple sclerosis? Brain 131, 1681-1683 (2008).

51. Ponath, G., Park, C. \& Pitt, D. The role of astrocytes in multiple sclerosis. Front. Immunol. 9, 217 (2018).

52. Lassmann, H. Pathogenic mechanisms associated with different clinical courses of multiple sclerosis. Front. Immunol. 9, 3116 (2019).

53. Machado-Santos, J. et al. The compartmentalized inflammatory response in the multiple sclerosis brain is composed of tissue-resident CD8 + T lymphocytes and B cells. Brain 141, 2066-2082 (2018).

54. ten Brinke, A. et al. Ways forward for tolerance-inducing cellular therapies-an afactt perspective. Front. Immunol. 10, 181 (2019).

55. Nutt, S. L. \& Chopin, M. Transcriptional networks driving dendritic cell differentiation and function. Immunity 52, 942-956 (2020)

56. Morelli, A. E. \& Thomson, A. W. Tolerogenic dendritic cells and the quest for transplant tolerance. Nat. Rev. Immunol. 7, 610-621 (2007).

57. Suwandi, J. S., Nikolic, T. \& Roep, B. O. Translating mechanism of regulatory action of tolerogenic dendritic cells to monitoring endpoints in clinical trials. Front. Immunol. 8, 22 (2017).

58. Kim, S. H., Jung, H. H. \& Lee, C. K. Generation, characteristics and clinical trials of ex vivo generated tolerogenic dendritic cells. Yonsei Med. J. Yonsei Univ. Coll. Med. 59, 807-815 (2018)

59. Van Brussel, I. et al. Tolerogenic dendritic cell vaccines to treat autoimmune diseases: Can the unattainable dream turn into reality? Autoimmun. Rev. 13, 138-150 (2014).

60. Zhou, Y. et al. Regulatory dendritic cells induced by K313 display anti-inflammatory properties and ameliorate experimental autoimmune encephalitis in mice. Front. Pharmacol. 10, 1579 (2020).

61. Derdelinckx, J. et al. Clinical and immunological control of experimental autoimmune encephalomyelitis by tolerogenic dendritic cells loaded with MOGencoding mRNA. J. Neuroinflammation. 16, 167 (2019).

62. Zhou, F., Zhang, G. X. \& Rostami, A. LPS-treated bone marrow-derived dendritic cells induce immune tolerance through modulating differentiation of CD4+ regulatory $\mathrm{T}$ cell subpopulations mediated by $3 \mathrm{G} 11$ and CD127. Immunol. Res. 65, 630-638 (2017)

63. Zhou, F., Zhang, G. X. \& Rostami, A. Apoptotic cell-treated dendritic cells induce immune tolerance by specifically inhibiting development of CD4+effector memory T cells. Immunol. Res. 64, 73-81 (2016).

64. Xie, Z. et al. 1,25-dihydroxyvitamin D3-induced dendritic cells suppress experimental autoimmune encephalomyelitis by increasing proportions of the regulatory lymphocytes and reducing $\mathrm{T}$ helper type 1 and type 17 cells. Immunology 152, 414-424 (2017).

65. Li, C. H. et al. Dendritic cells, engineered to overexpress 25 -hydroxyvitamin D 1a-hydroxylase and pulsed with a myelin antigen, provide myelin-specific suppression of ongoing experimental allergic encephalomyelitis. FASEB J. 31, 2996-3006 (2017).

66. Mansilla, M. J. et al. Cryopreserved vitamin D3-tolerogenic dendritic cells pulsed with autoantigens as a potential therapy for multiple sclerosis patients. $J \mathrm{Neu}$ roinflammation. 13, 113 (2016).

67. Mansilla, M. J. et al. Beneficial effect of tolerogenic dendritic cells pulsed with MOG autoantigen in experimental autoimmune encephalomyelitis. CNS Neurosci. Ther. 21, 222-230 (2015).

68. Thome, R. et al. Chloroquine-treated dendritic cells require STAT1 signaling for their tolerogenic activity. Eur. J. Immunol. 48, 1228-1234 (2018).

69. Zhou, Y. et al. Tolerogenic dendritic cells generated with tofacitinib ameliorate experimental autoimmune encephalomyelitis through modulation of Th17/Treg balance. J. Immunol. Res. 2016, 5021537 (2016).

70. Zhou, Y. et al. Tolerogenic dendritic cells induced by BD750 ameliorate proinflammatory $T$ cell responses and experimental autoimmune encephalitis in mice. Mol. Med. 23, 204-214 (2017).

71. Haller, S. et al. Interleukin-35-producing CD8a + dendritic cells acquire a tolerogenic state and regulate T cell function. Front Immunol. 8, 98 (2017).

72. Grau-López, L. et al. Specific T-cell proliferation to myelin peptides in relapsingremitting multiple sclerosis. Eur. J. Neurol. 18, 1101-1104 (2011).

73. Lutterotti, A. et al. Antigen-specific tolerance by autologous myelin peptidecoupled cells: A phase 1 trial in multiple sclerosis. Sci. Transl. Med. 5, 188ra75 (2013).

74. Zubizarreta, I. et al. Immune tolerance in multiple sclerosis and neuromyelitis optica with peptide-loaded tolerogenic dendritic cells in a phase $1 \mathrm{~b}$ trial. Proc. Natl Acad. Sci. USA. 116, 8463-8470 (2019).

75. Lee, W. P. et al. Immunomodulatory effects of 1,25-dihydroxyvitamin D3 on dendritic cells promote induction of $\mathrm{T}$ cell hyporesponsiveness to myelinderived antigens. J. Immunol. Res. 2016, 5392623 (2016). 
76. Jauregui-Amezaga, A. et al. Intraperitoneal administration of autologous tolerogenic dendritic cells for refractory Crohn's disease: a phase I study. J. Crohns Colitis 9, 1071-1078 (2015).

77. Giannoukakis, N., Phillips, B., Finegold, D., Harnaha, J. \& Trucco, M. Phase I (safety) study of autologous tolerogenic dendritic cells in type 1 diabetic patients. Diabetes Care. 34, 2026-2032 (2011).

78. Benham, H. et al. Citrullinated peptide dendritic cell immunotherapy in HLA risk genotype-positive rheumatoid arthritis patients. Sci. Transl. Med. 7, 290ra87 (2015).

79. Bell, G. M. et al. Autologous tolerogenic dendritic cells for rheumatoid and inflammatory arthritis. Ann. Rheum. Dis. 76, 227-234 (2017).

80. Moreau, A. et al. Generation and in vivo evaluation of IL10-treated dendritic cells in a nonhuman primate model of AAV-based gene transfer. Mol. Ther. Methods Clin. Dev. 1, 14028 (2014).

81. Willekens, B. et al. Tolerogenic dendritic cell-based treatment for multiple sclerosis (MS): a harmonised study protocol for two phase I clinical trials comparing intradermal and intranodal cell administration. BMJ Open. 9, e030309 (2019).

82. Raïch-Regué, D. et al. Stable antigen-specific T-cell hyporesponsiveness induced by tolerogenic dendritic cells from multiple sclerosis patients. Eur. J. Immunol. 42, 771-782 (2012).

83. Navarro-Barriuso, J. et al. Vitamin D3-induced tolerogenic dendritic cells modulate the transcriptomic profile of $\mathrm{T} \mathrm{CD} 4+$ cells towards a functional hyporesponsiveness. Front. Immunol. 11, 599623 (2020)

84. Anderson, A. E. et al. Tolerogenic dendritic cells generated with dexamethasone and vitamin D3 regulate rheumatoid arthritis CD4 + T cells partly via transforming growth factor- $\beta 1$. Clin. Exp. Immunol. 187, 113-123 (2017).

85. Marín, E., Cuturi, M. C. \& Moreau, A. Tolerogenic dendritic cells in solid organ transplantation: Where do we stand? Front. Immunol. 9, 274 (2018).

86. Djedovic, N. et al. Ethyl pyruvate induces tolerogenic dendritic cells. Front. Immunol. 10, 157 (2019).

87. Navarro-Barriuso, J. et al. MAP7 and MUCL1 are biomarkers of Vitamin D3induced tolerogenic dendritic cells in multiple sclerosis patients. Front. Immunol. 10, 1251 (2019).

88. Chung, D. J. et al. Indoleamine 2,3-dioxygenase-expressing mature human monocyte-derived dendritic cells expand potent autologous regulatory $\mathrm{T}$ cells. Blood 114, 555-563 (2009).

89. Navarro-Barriuso, J., Mansilla, M. J. \& Martínez-Cáceres, E. M. Searching for the Transcriptomic Signature of Immune Tolerance Induction-Biomarkers of Safety and Functionality for Tolerogenic Dendritic Cells and Regulatory Macrophages. Front Immunol. 9, 2062 (2018).

90. Voigtländer, C. et al. Dendritic cells matured with TNF can be further activated in vitro and after subcutaneous injection in vivo which converts their tolerogenicity into immunogenicity. J. Immunother. 29, 407-415 (2006).

91. Lee, H. W., Gangadaran, P., Kalimuthu, S. \& Ahn, B. C. Advances in molecular imaging strategies for in vivo tracking of immune cells. BioMed Res. Int. 2016, 1946585 (2016)

92. Gliwiński, M., Iwaszkiewicz-Grześ, D. \& Trzonkowski, P. Cell-based therapies with T regulatory cells. BioDrugs 31, 335-347 (2017).

93. Zhang, X. et al. IL-10 is involved in the suppression of experimental autoimmune encephalomyelitis by $\mathrm{CD} 25+\mathrm{CD} 4+$ regulatory $\mathrm{T}$ cells. Int Immunol. 16, 249-256 (2004).

94. Chwojnicki, K. et al. Intrathecal administration of $\mathrm{CD}^{+}{ }^{+} \mathrm{CD} 25$ high $\mathrm{CD} 127^{-}$foxp3 $^{+}$ regulatory $T$ cells slows progression of multiple sclerosis-Results of phase I study. Biodrugs. 35, 47-60 (2021).

95. Feger, U. et al. Increased frequency of CD4 $+C D 25+$ regulatory $T$ cells in the cerebrospinal fluid but not in the blood of multiple sclerosis patients. Clin. Exp. Immunol. 147, 412-418 (2007).

96. Sharabi, A. et al. Regulatory T cells in the treatment of disease. Nat. Rev. Drug Discov. 17, 823-844 (2018).

97. Marek-Trzonkowska, N. et al. Factors affecting long-term efficacy of T regulatory cell-based therapy in type 1 diabetes. J. Transl. Med. 14, 332 (2016).

98. Long, S. A. et al. Rapamycin/IL-2 combination therapy in patients with type diabetes augments Tregs yet transiently impairs $\beta$-cell function. Diabetes $\mathbf{6 1}$, 2340-2348 (2012)

99. Schmitz, R. et al. Kidney Transplantation using Alemtuzumab, Belatacept and Sirolimus-Five-Year Follow-up. Am. J. Transplant. 20, 3609-3619 (2020).

100. Sawitzki, B. et al. Regulatory cell therapy in kidney transplantation (The ONE Study): a harmonised design and analysis of seven non-randomised, single-arm, phase 1/2A trials. Lancet. 395, 1627-1639 (2020).

101. Stephens, L. A., Malpass, K. H., Anderton, S. M. \& Curing, C. N. S. autoimmune disease with myelin-reactive Foxp3+ Treg. Eur. J. Immunol 39, 1108-1117 (2009).

102. Fransson, M. et al. CAR/FoxP3-engineered T regulatory cells target the CNS and suppress EAE upon intranasal delivery. J. Neuroinflamm 9, 112 (2012).

103. Niedbala, W. et al. Nitric Oxide-Induced Regulatory T Cells Inhibit Th17 but Not Th1 Cell Differentiation and Function. J. Immunol. 191, 164-170 (2013).
104. Malviya, M., Saoudi, A., Bauer, J., Fillatreau, S. \& Liblau, R. Treatment of experimental autoimmune encephalomyelitis with engineered bi-specific Foxp3+ regulatory CD4 + T cells. J. Autoimmun. 108, 102401 (2020).

105. Verhagen, J., Gabryšová, L., Shepard, E. R. \& Wraith, D. C. CTLA-4 modulates the differentiation of inducible foxp3+treg cells but IL-10 mediates their function in experimental autoimmune encephalomyelitis. PLOS ONE 9, e108023 (2014).

106. $\mathrm{Yu}, \mathrm{P}$. et al. Specific $\mathrm{T}$ regulatory cells display broad suppressive functions against experimental allergic encephalomyelitis upon activation with cognate antigen. J. Immunol. 174, 6772-6780 (2005).

107. Zhang, H., Podojil, J. R., Chang, J., Luo, X. \& Miller, S. D. TGF- $\beta$-induced myelin peptide-specific regulatory $T$ cells mediate antigen-specific suppression of induction of experimental autoimmune encephalomyelitis. J. Immunol. 184, 6629-6636 (2010).

108. Chen, M.-L., Yan, B.-S., Bando, Y., Kuchroo, V. K. \& Weiner, H. L. Latencyassociated peptide identifies a novel $C D 4+C D 25+$ regulatory $T$ cell subset with TGF $\beta$-mediated function and enhanced suppression of experimental autoimmune encephalomyelitis. J. Immunol. 180, 7327-7337 (2008).

109. Lüth, S. et al. Ectopic expression of neural autoantigen in mouse liver suppresses experimental autoimmune neuroinflammation by inducing antigen-specific Tregs. J. Clin. Investig. 118, 3403-3410 (2008).

110. McPherson, R. C., Turner, D. G., Mair, I., O'Connor, R. A. \& Anderton, S. M. T-bet expression by Foxp $3+\mathrm{T}$ regulatory cells is not essential for their suppressive function in CNS autoimmune disease or colitis. Front. Immunol. 6, 69 (2015).

111. Kim, Y. C. et al. Engineered MBP-specific human Tregs ameliorate MOG-induced EAE through IL-2-triggered inhibition of effector T cells. J. Autoimmun. 92, 77-86 (2018).

112. MacDonald, K. G. et al. Alloantigen-specific regulatory T cells generated with a chimeric antigen receptor. J. Clin. Investig. 126, 1413-1424 (2016).

113. Paweł, Ł., Trzonkowski, P. \& Chwojnicki, K. Multiple sclerosis-new therapeutic directions. Eur. J. Transl. Clin. Med. 2, 7-22 (2017).

114. Riedhammer, C. \& Weissert, R. Antigen presentation, autoantigens, and immune regulation in multiple sclerosis and other autoimmune diseases. Front. Immunol. 6, 322 (2015).

115. Boardman, D. A. et al. Expression of a chimeric antigen receptor specific for donor HLA class I enhances the potency of human regulatory T cells in preventing human skin transplant rejection. Am. J. Transplant. 17, 931-943 (2017).

116. Noyan, F. et al. Prevention of allograft rejection by use of regulatory $T$ cells with an MHC-specific chimeric antigen receptor. Am. J. Transplant. 17, 917-930 (2017).

117. Trzonkowski, P. et al. Hurdles in therapy with regulatory T cells. Sci. Transl. Med. 7, 304ps18 (2015)

118. Gliwiński, M. et al. Proinsulin-specific $T$ regulatory cells may control immune responses in type 1 diabetes: Implications for adoptive therapy. BMJ Open Diabetes Res Care. 8, e000873 (2020).

119. Hull, C. M. et al. Generation of human islet-specific regulatory T cells by TCR gene transfer. J. Autoimmun. 79, 63-73 (2017).

120. Friedenstein, A. J., Petrakova, K. V. \& Kurolesova, A. I. F. G. Heterotopic of bone marrow. Analysis of precursor cells for osteogenic and hematopoietic tissues. Transplantation 6, 230-247 (1968).

121. Caplan, A. I. Mesenchymal stem cells. J Orthop Res. 9, 641-650 (1991).

122. Dominici, M. et al. Minimal criteria for defining multipotent mesenchymal stromal cells. The International Society for Cellular Therapy position statement Cytotherapy 8, 315-317 (2006).

123. Zappia, E. et al. Mesenchymal stem cells ameliorate experimental autoimmune encephalomyelitis inducing T-cell anergy. Blood 106, 1755-1761 (2005).

124. Yanwu, Y., Meiling, G., Yunxia, Z., Qiukui, H. \& Birong, D. Mesenchymal stem cells in experimental autoimmune encephalomyelitis model of multiple sclerosis: a systematic review and meta-analysis. Mult. Scler. Relat. Disord 44, 102200 (2020).

125. Mansoor, $\mathrm{H}$. et al. Current trends and future perspective of mesenchymal stem cells and exosomes in corneal diseases. Int. J. Mol. Sci. 20, 2853 (2019).

126. Wang, S., Qu, X. \& Zhao, R. C. Clinical applications of mesenchymal stem cells. J. Hematol. Oncol. 5, 19 (2012).

127. Li, L. et al. Exosomes derived from mesenchymal stem cells ameliorate renal ischemic-reperfusion injury through inhibiting inflammation and cell apoptosis. Front. Med. 6, 269 (2019).

128. Di Nicola, M. et al. Human bone marrow stromal cells suppress T-lymphocyte proliferation induced by cellular or nonspecific mitogenic stimuli. Blood $\mathbf{9 9}$, 3838-3843 (2002)

129. Aggarwal, S. \& Pittenger, M. F. Human mesenchymal stem cells modulate allogeneic immune cell responses. Blood 105, 1815-1822 (2005).

130. English, K. et al. Cell contact, prostaglandin E2 and transforming growth factor beta 1 play non-redundant roles in human mesenchymal stem cell induction of CD4 + CD25Highforkhead box P3 + regulatory T cells. Clin. Exp. Immunol 156, 149-160 (2009).

131. Augello, A. et al. Bone marrow mesenchymal progenitor cells inhibit lymphocyte proliferation by activation of the programmed death 1 pathway. Eur. J. Immunol. 35, 1482-1490 (2005). 
132. Corcione, A. et al. Human mesenchymal stem cells modulate B-cell functions. Blood 107, 367-372 (2006).

133. Asari, S. et al. Mesenchymal stem cells suppress B-cell terminal differentiation. Exp. Hematol. 37, 604-615 (2009).

134. Sotiropoulou, P. A., Perez, S. A., Gritzapis, A. D., Baxevanis, C. N. \& Papamichail, M. Interactions between human mesenchymal stem cells and natural killer cells. Stem Cells 24, 74-85 (2006).

135. Spaggiari, G. M., Capobianco, A., Becchetti, S., Mingari, M. C. \& Moretta, L. Mesenchymal stem cell-natural killer cell interactions: evidence that activated NK cells are capable of killing MSCs, whereas MSCs can inhibit IL-2-induced NKcell proliferation. Blood 107, 1484-1490 (2006).

136. Chen, L. et al. Effects of human mesenchymal stem cells on the differentiation of dendritic cells from CD34 + cells. Stem Cells Dev 16, 719-731 (2007).

137. Li, Z. et al. Exosomes derived from mesenchymal stem cells attenuate inflammation and demyelination of the central nervous system in EAE rats by regulating the polarization of microglia. Int Immunopharmacol. 67, 268-280 (2019).

138. Devine, S. M., Cobbs, C., Jennings, M., Bartholomew, A. \& Hoffman, R. Mesenchymal stem cells distribute to a wide range of tissues following systemic infusion into nonhuman primates. Blood 101, 2999-3001 (2003).

139. Li, Y. et al. Human marrow stromal cell therapy for stroke in rat: neurotrophins and functional recovery. Neurology 59, 514-523 (2002).

140. Munoz, J. R., Stoutenger, B. R., Robinson, A. P., Spees, J. L. \& Prockop, D. J. Human stem/progenitor cells from bone marrow promote neurogenesis of endogenous neural stem cells in the hippocampus of mice. Proc. Natl Acad. Sci. USA. 102, 18171-18176 (2005).

141. Rivera, F. J. et al. Mesenchymal stem cells instruct oligodendrogenic fate decision on adult neural stem cells. Stem Cells 24, 2209-2219 (2006).

142. Uccelli, A. et al. MEsenchymal StEm cells for Multiple Sclerosis (MESEMS): A randomized, double blind, cross-over phase $1 / I$ clinical trial with autologous mesenchymal stem cells for the therapy of multiple sclerosis. Trials. 20, 263 (2019).

143. Llufriu, S. et al. Randomized placebo-controlled phase II trial of autologous mesenchymal stem cells in multiple sclerosis. PLoS ONE. 9, e113936 (2014).

144. Cohen, J. A. et al. Pilot trial of intravenous autologous culture-expanded mesenchymal stem cell transplantation in multiple sclerosis. Mult. Scler. J. 24, 501-511 (2018)

145. Connick, P. et al. The mesenchymal stem cells in multiple sclerosis (MSCIMS) trial protocol and baseline cohort characteristics: an open-label pre-test: Post-test study with blinded outcome assessments. Trials. 12, 62 (2011).

146. Connick, P. et al. Autologous mesenchymal stem cells for the treatment of secondary progressive multiple sclerosis: An open-label phase 2a proof-ofconcept study. Lancet Neurol. 11, 150-156 (2012).

147. Karussis, D. et al. Safety and immunological effects of mesenchymal stem cell transplantation in patients with multiple sclerosis and amyotrophic lateral sclerosis. Arch. Neurol. 67, 1187-1194 (2010).

148. Rice, C. M. et al. Repeat infusion of autologous bone marrow cells in multiple sclerosis: Protocol for a phase i extension study (SIAMMS-II). BMJ Open. 5, e009090 (2015).

149. Rice, C. M. et al. Assessment of bone marrow-derived Cellular Therapy in progressive Multiple Sclerosis (ACTiMuS): Study protocol for a randomised controlled trial. Trials. 16, 463 (2015).

150. Fernández, O. et al. Adipose-derived mesenchymal stem cells (AdMSC) for the treatment of secondary-progressive multiple sclerosis: A triple blinded, placebo controlled, randomized phase I/II safety and feasibility study. PLOS ONE. 13 , e0195891 (2018).

151. Riordan, N. H. et al. Clinical feasibility of umbilical cord tissue-derived mesenchymal stem cells in the treatment of multiple sclerosis. J. Transl. Med. 16, 57 (2018).

152. Sarkar, P. et al. Reduced neuroprotective potential of the mesenchymal stromal cell secretome with ex vivo expansion, age and progressive multiple sclerosis. Cytotherapy 20, 21-28 (2018).

153. Massey, J. C., Sutton, I. J., Ma, D. D. F. \& Moore, J. J. Regenerating immunotolerance in multiple sclerosis with autologous hematopoietic stem cell transplant. Front. Immunol. 9, 410 (2018).

154. Fassas, A. et al. Peripheral blood stem cell transplantation in the treatment of progressive multiple sclerosis: First results of a pilot study. Bone Marrow Transplant. 20, 631-638 (1997).

155. Arruda, L. C. M. et al. Immunological correlates of favorable long-term clinical outcome in multiple sclerosis patients after autologous hematopoietic stem cell transplantation. Clin. Immunol. 169, 47-57 (2016).

156. Cuascut, F. X. \& Hutton, G. J. Stem cell-based therapies for multiple sclerosis: current perspectives. Biomedicines. 7, 26 (2019).

157. Muraro, P. A. et al. Autologous haematopoietic stem cell transplantation for treatment of multiple sclerosis. Nat. Rev. Neurol. 13, 391-405 (2017).
158. Pluchino, S., Smith, J. A. \& Peruzzotti-Jametti, L. Promises and limitations of neural stem cell therapies for progressive multiple sclerosis. Trend. Mol. Med. 26, 898-912 (2020).

159. Ben-Nun, A. \& Wekerle, H. C. I. Vaccination against autoimmune encephalomyelitis with T-lymphocyte line cells reactive against myelin basic protein. Nature $\mathbf{2 9 2}, 60$ 61 (1981).

160. Zhang, J., Medaer, R., Stinissen, P., Hafler, D. \& Raus, J. MHC-restricted depletion of human myelin basic protein-reactive $T$ cells by $T$ cell vaccination. Science. 261, 1451-1454 (1993).

161. Medaer, R., Stinissen, P., Raus, J., Zhang, J. \& Truyen, L. Depletion of myelin-basicprotein autoreactive $\mathrm{T}$ cells by $\mathrm{T}$-cell vaccination: pilot trial in multiple sclerosis. Lancet. 346, 807-808 (1995).

162. Correale J., et al. T cell vaccination in secondary progressive multiple sclerosis. J. Neuroimmunol. 107, (2000)

163. Karussis, D. et al. T cell vaccination benefits relapsing progressive multiple sclerosis patients: a randomized, double-blind clinical trial. PLOS ONE. 7, e50478 (2012).

164. Loftus, B. et al. Autologous attenuated T-cell vaccine (Tovaxin ${ }^{\circledR}$ ) dose escalation in multiple sclerosis relapsing-remitting and secondary progressive patients nonresponsive to approved immunomodulatory therapies. Clin. Immunol. 131, 202-215 (2009).

165. Vandenbark, A. A. \& Abulafia-Lapid, R. Autologous T-cell vaccination for multiple sclerosis: a perspective on progress. BioDrugs 22, 265-273 (2008).

166. Zhang, J. Z. et al. T cell vaccination in multiple sclerosis: results of a preliminary study. J. Neurol. 249, 212-218 (2002).

167. Van Der $A a, A$. et al. T cell vaccination in multiple sclerosis patients with autologous CSF-derived activated T cells: results from a pilot study. Clin. Exp. Immunol. 131, 155-168 (2003).

168. Hafler, D., Iruncohen, D. A., Benjamin, D. \& Weiner, H. L. T cell vaccination in multiple sclerosis: a preliminary report. Clin. Immunol. Immunopathol. 62, 307-313 (1992).

169. Achiron, A. et al. T cell vaccination in multiple sclerosis relapsing-remitting nonresponders patients. Clin. Immunol. 113, 155-160 (2004).

170. Fox, E. et al. A randomized clinical trial of autologous T-cell therapy in multiple sclerosis: Subset analysis and implications for trial design. Mult. Scler. J. 18, 843852 (2012).

171. Ezzelarab, M. B. et al. Regulatory dendritic cell infusion prolongs kidney allograft survival in nonhuman primates. Am. J. Transplant. 13, 1989-2005 (2013).

172. Koreth, J. et al. Interleukin-2 and regulatory T cells in graft-versus-host disease abstract from the divisions of hematologic malig-nancies. N. Engl. J. Med. 22, 2055-2066 (2011).

173. Zielinski, M. et al. Combined immunotherapy with $\mathrm{T}$ regulatory cells and antiCD20 antibody prolongs survival of pancreatic islets in type 1 diabetes. Diabetes 69, 66-LB (2020).

174. Phillips, B. E., Garciafigueroa, Y., Engman, C., Trucco, M. \& Giannoukakis, N. Tolerogenic dendritic cells and T-regulatory cells at the clinical trials crossroad for the treatment of autoimmune disease; emphasis on type 1 diabetes therapy. Front. Immunol. 10, 148 (2019).

175. Li, R. et al. Synergistic suppression of autoimmune arthritis through concurrent treatment with tolerogenic DC and MSC. Sci. Rep. 7, 43188 (2017).

176. Lee, E. S. et al. Adoptive transfer of treg cells combined with mesenchymal stem cells facilitates repopulation of endogenous treg cells in a murine acute GVHD model. PLOS ONE. 10, e0138846 (2015).

177. Lord, P. et al. Minimum information about tolerogenic antigen-presenting cells (MITAP): a first step towards reproducibility and standardisation of cellular therapies. PeerJ. 4, e2300 (2016).

178. Fuchs, A. et al. Minimum information about $T$ regulatory cells: a step toward reproducibility and standardization. Front. Immunol. 8, 1844 (2018).

Open Access This article is licensed under a Creative Commons Attribution 4.0 International License, which permits use, sharing, adaptation, distribution and reproduction in any medium or format, as long as you give appropriate credit to the original author(s) and the source, provide a link to the Creative Commons license, and indicate if changes were made. The images or other third party material in this article are included in the article's Creative Commons license, unless indicated otherwise in a credit line to the material. If material is not included in the article's Creative Commons license and your intended use is not permitted by statutory regulation or exceeds the permitted use, you will need to obtain permission directly from the copyright holder. To view a copy of this license, visit http://creativecommons. org/licenses/by/4.0/.

(c) The Author(s) 2021 